\title{
Sufficient conditions for polynomial asymptotic behaviour of the stochastic pantograph equation
}

\author{
John A. D. Appleby ${ }^{\bowtie 1}$ and Evelyn Buckwar ${ }^{* 2}$ \\ ${ }^{1}$ School of Mathematical Sciences, Dublin City University, Glasnevin, Dublin 9, Ireland \\ ${ }^{2}$ Johannes Kepler University, Institute for Stochastics, Altenbergerstrasse 69, 4040 Linz, Austria
}

Appeared 11 August 2016

Communicated by Tibor Krisztin

\begin{abstract}
This paper studies the asymptotic growth and decay properties of solutions of the stochastic pantograph equation with multiplicative noise. We give sufficient conditions on the parameters for solutions to grow at a polynomial rate in $p$-th mean and in the almost sure sense. Under stronger conditions the solutions decay to zero with a polynomial rate in $p$-th mean and in the almost sure sense. When polynomial bounds cannot be achieved, we show for a different set of parameters that exponential growth bounds of solutions in $p$-th mean and an almost sure sense can be obtained. Analogous results are established for pantograph equations with several delays, and for general finite dimensional equations.
\end{abstract}

Keywords: stochastic pantograph equation, asymptotic stability, stochastic delay differential equations, unbounded delay, polynomial asymptotic stability, decay rates.

2010 Mathematics Subject Classification: 60H10, 34K20, 34K50.

\section{Authors' note}

Much of the contents of the following paper was written in 2003, and a preprint of the work has been available online under this title since that date [5]. To the best of the authors' knowledge in 2003, this work was the first concerning the asymptotic behaviour of stochastic equations with proportional (or indeed unbounded point) delay. Such stochastic proportional delay equations are often called stochastic pantograph equations. The paper did not find a ready home at the time, but subsequently has steadily attracted citations through its online preprint incarnation. These are included in the bibliography below $[3,6,19,23,24,27,33,44,45$, 48-52,55,56]. Since a number of other works quote [5], we feel it best that the paper be subject to formal review, and as the first author's introduction to the subject came through a paper in the EJQTDE [38], we felt it fitting, after a long (but not unbounded) delay, to submit a revised version of it here.

In fact, asymptotic analysis of stochastic equations with unbounded delay of this type have been the subject of many works, as can be confirmed by investigation of citation databases.

\footnotetext{
${ }^{\bowtie}$ Corresponding author. Email: john.appleby@dcu.ie

${ }^{*}$ Email: Evelyn.Buckwar@jku.at
} 
Results giving general asymptotic rates, rather than the polynomial behaviour recorded here, have been extended since 2003 to deal with uncertain neural networks, as for example can be seen in [36].

We are also grateful for the support of American Institute of Mathematics to enable us to make the appropriate revisions and updates to the work.

\section{Introduction}

In this paper we shall study the asymptotic behaviour of the stochastic pantograph equation

$$
\begin{aligned}
d X(t) & =\{a X(t)+b X(q t)\} d t+\{\sigma X(t)+\rho X(q t)\} d B(t), \quad t \geq 0, \\
X(0) & =X_{0} .
\end{aligned}
$$

We assume that $a, b, \sigma, \rho$ are real constants and $q \in(0,1)$. The process $(B(t))_{t \geq 0}$ is a standard one dimensional Wiener process, given on a filtered probability space $(\Omega, \mathcal{F}, \mathbb{P})$. The filtration is the natural filtration of $B$. The initial value $X_{0}$ satisfies $\mathbb{E}\left(\left|X_{0}\right|^{2}\right)<\infty$ and is independent of $B$.

We denote a solution of (2.1), starting at 0 and with initial condition $X_{0}$ by $\left(X\left(t ; 0, X_{0}\right)\right)_{t \geq 0}$. By [14] there exists a path-wise unique strong solution $\left(X\left(t ; 0, X_{0}\right)\right)_{t \geq 0}$ of (2.1).

Equation (2.1) is a generalisation of the deterministic pantograph equation

$$
x^{\prime}(t)=\bar{a} x(t)+\bar{b} x(q t), \quad t \geq 0, \quad x(0)=x_{0}, \quad q \in(0,1),
$$

in which it is conventional to take $x^{\prime}(t)$ to denote the right-hand derivative of $x$.

Since $q t<t$ when $t \geq 0$, equations (2.1) and (2.2) are differential equations with time lag. The quantity $\tau(t)=t-q t$ in the delayed argument of $x(t-\tau(t))$ will be called the (variable) lag. We note that the argument $q t$ satisfies $q t \rightarrow \infty$ as $t \rightarrow \infty$ but the lag is unbounded, that is $t-q t \rightarrow \infty$ as $t \rightarrow \infty$. In the literature equations like (2.1) and (2.2) are also termed (stochastic) delay, retarded or functional differential equations.

Equation (2.2) and its generalisations possess a wide range of applications. Equation (2.2) arises, for example, in the analysis of the dynamics of an overhead current collection system for an electric locomotive or in the problem of a one-dimensional wave motion, such as that due to small vertical displacements of a stretched string under gravity, caused by an applied force which moves along the string ([20] and [47]). Existence, uniqueness and asymptotic properties of the solution of (2.2) and its generalisations have been considered in [17, 26, 29, 38]. Equation (2.2) can be used as a paradigm for the construction of numerical schemes for functional differential equations with unbounded lag, $c f$. $[15,25,37]$ (we do not attempt to give a complete list of references here).

A wealth of literature now exists on the non-exponential (general) rates of decay to equilibrium of solutions of differential and functional differential equations, both for deterministic and stochastic equations. Three types of equations which exhibit such general (nonexponential) rates of decay have attracted much attention. These are

(i) non-autonomous perturbations or forcing terms added to linear or near-linear problems (such as quasi-linear, or semi-linear equations);

(ii) nonlinear equations (which have no linear, or linearisable terms near equilibrium), giving rise to weak exponential asymptotic stability; 
(iii) certain types of linear equations with unbounded delay.

In the deterministic theory, all three mechanisms have been studied extensively. For stochastic differential equations, and functional differential equations, several authors have obtained results in categories (i), (ii), but comparatively few results have been established in category (iii). We will briefly review the literature on non-exponential stability of solutions of SDEs and SFDEs in categories (i), (ii), and allude to the relevant theory for deterministic problems in category (iii).

An important subclass of non-exponential behaviour is the so-called polynomial asymptotic stability, where the rate of decay is bounded by a polynomial with negative exponent, in either a $p$-th mean or almost sure sense. This type of stability has been studied in Mao [40,41], Mao and Liu [35], for stochastic differential equations and stochastic functional differential equations with bounded delay, principally for problems of type (i). More general rates of decay than polynomial are considered in these papers, but in most cases, it is the non-exponential nature of non-autonomous perturbations, that gives rise to the non-exponential decay rates of solutions.

The problem (ii) has been investigated for stochastic differential equations in Zhang and Tsoi [53,54], and Liu [34] with state-dependent noise and in Appleby and Mackey [7] and Appleby and Patterson [8] for state independent noise. In these papers, it is principally the nonlinear form of the equation close to equilibrium that gives rise to the slow decay of the solution to equilibrium, rather than non-autonomous time-dependent terms (for deterministic functional differential equations, results of this type can be found in Krisztin [30], and Haddock and Krisztin [21,22]; general decay rates for deterministic problems of types (i), (ii) are considered in Caraballo [16]).

The third mechanism (iii) by which SFDEs can approach equilibrium more slowly than exponentially has been less studied, and motivates the material in this paper. To this end, we briefly reprise the convergence properties of linear autonomous deterministic functional differential equations with bounded delay and unbounded delay. Bounded delay equations of this type must converge to zero exponentially fast, if the equilibrium is uniformly asymptotically stable. However, convergence to equilibrium need not be at an exponential rate for equations with unbounded delay. For example, for a linear convolution Volterra integrodifferential equation, Murakami showed in [46] that the exponential asymptotic stability of the zero solution requires a type of exponential decay criterion on the kernel, Appleby and Reynolds [9] have determined exact sub-exponential decay rates on the solutions of Volterra equations, while Kato and McLeod [29] have shown that solutions of the linear equation (2.2) can converge to zero at a slower than exponential (polynomial) rate.

In contrast to categories (i), (ii) for SDEs and SFDEs, less is known regarding the nonexponential asymptotic behaviour of linear stochastic functional differential equations with unbounded delay, although it has been shown in $[2,4,10-12]$ that solutions of linear convolution Itô-Volterra equations can converge to equilibrium at a non-exponential rate.

In this paper, we show that, in common with the deterministic pantograph equation studied in [29], solutions of the stochastic pantograph equation (2.1) can be bounded by polynomials in both a $p$-th mean and almost sure sense, and, for values of the parameters $a, b, \sigma, \rho, q$, we establish polynomial asymptotic stability in these senses. Furthermore, it appears, in common with the deterministic pantograph equation, that the polynomial asymptotic behaviour is determined only by the values of the parameters associated with the non-delay terms. We also observe, when the noise intensities $\sigma, \rho$ are small, that the polynomial asymptotic behaviour of the stochastic problem can be inferred from that of the corresponding deterministic equation. 
Our analysis involves obtaining estimates on the second mean of the solution of (2.1) using comparison principle arguments (as in [14]), and then using these estimates to obtain upper bounds on the solution in an almost sure sense, using an idea of Mao [42].

The article is organised as follows: in Section 3 we give the definitions of the asymptotic behaviour of solutions that we want to discuss and we state the relevant properties of the deterministic pantograph equation.

In Section 4 sufficient conditions are given for which the solution process is bounded asymptotically by polynomials, in a first mean and a mean-square sense, as well as in an almost sure sense. On a restriction of this parameter set, we show that the equilibrium solution is asymptotically stable in a $p$-th mean sense $(p=1,2)$ or almost surely, with the decay rate bounded above by a polynomial.

In Section 5 we consider unstable solutions of (2.1) and parameter regions in which the polynomial boundedness of these solutions has not been established. We prove that all such solutions are bounded by increasing exponentials in the first mean and in mean square and almost surely.

In the penultimate section of the paper, we show that the analysis of the scalar stochastic pantograph equation with one proportional delay extends to equations with arbitrarily many proportional delays, and also to finite dimensional analogues of (2.1). The final section discusses some related problems; an Appendix contains several technical results.

\section{Preliminary results}

We state the definitions for the asymptotic behaviour in this section. We follow the definition given in Mao $[39,40]$.

\subsection{Definitions of asymptotic behaviour}

First we define the notions of asymptotic growth that we consider in this article. Notice that

$$
X\left(t ; 0, X_{0}\right)=X_{0} X(t ; 0,1), \quad t \geq 0 .
$$

Therefore, because $X_{0}$ is independent of $B$, bounds on the $p$-th moment of $X\left(t ; 0, X_{0}\right)$ will be linear in $\mathbb{E}\left(\left|X_{0}\right|^{p}\right)$. This fact is reflected in the definition below.

Definition 3.1. Let $\left(X\left(t ; 0, X_{0}\right)\right)_{t \geq 0}$ be the unique solution of the SDDE (2.1) and $p>0$. We say that the solution is

(1) globally polynomially bounded in $p$-th mean, if there exist constants $C>0, \alpha_{1} \in \mathbb{R}$, such that

$$
\mathbb{E}\left(\left|X\left(t ; 0, X_{0}\right)\right|^{p}\right) \leq C \mathbb{E}\left(\left|X_{0}\right|^{p}\right)(1+t)^{\alpha_{1}}, \quad t \geq 0 ;
$$

(2) almost surely globally polynomially bounded, if there exists a constant $\alpha_{2} \in \mathbb{R}$ such that

$$
\limsup _{t \rightarrow \infty} \frac{\log \left|X\left(t ; 0, X_{0}\right)\right|}{\log t} \leq \alpha_{2}, \quad \text { almost surely }
$$

(3) globally exponentially bounded in $p$-th mean, if there exist constants $C>0, \alpha_{3} \in \mathbb{R}$, such that

$$
\mathbb{E}\left(\left|X\left(t ; 0, X_{0}\right)\right|^{p}\right) \leq C \mathbb{E}\left(\left|X_{0}\right|^{p}\right) e^{\alpha_{3} t}, \quad t \geq 0 ;
$$


(4) almost surely globally exponentially bounded, if there exists a constant $\alpha_{4} \in \mathbb{R}$ such that

$$
\limsup _{t \rightarrow \infty} \frac{\log \left|X\left(t ; 0, X_{0}\right)\right|}{t} \leq \alpha_{4}, \quad \text { almost surely. }
$$

In the case that (1) holds for some $\alpha_{1} \geq 0$, we have that (3) holds for some $\alpha_{3} \geq 0$, and if (2) holds for some $\alpha_{2} \geq 0$, then (4) holds for some $\alpha_{4} \geq 0$. Also, if there is $\alpha_{3} \leq 0$ such that (3) holds, then there is $\alpha_{1} \leq 0$ such that (1) holds, and the existence of an $\alpha_{4} \leq 0$ such that (4) holds implies the existence of an $\alpha_{2} \leq 0$ such that (2) holds.

We stop to justify and clarify some aspects of our definition.

First, we note that the constants $\alpha_{i}$ for $i=1, \ldots, 4$ in each of (1)-(4) are independent of $X_{0}$ by dint of (3.1).

Second, although we choose to consider bounds in (3.2a) and (3.2c) for all $t \geq 0$, it is equivalent to start with a definition on which such bounds hold for $t \geq T$, for some $T>0$.

To prove this second remark, we concentrate on the polynomial case, noting that the situation is similar in the exponential one. Clearly, if we take as our starting point in lieu of (3.2a) a polynomial estimate that holds for all $t \geq T$ (where $T$ is sufficiently large), we may assume

$$
\mathbb{E}\left[|X(t ; 0,1)|^{p}\right] \leq C^{\prime}(1+t)^{\alpha}, \quad t \geq T,
$$

where $C^{\prime}>0$ and $\alpha$ are independent of $X_{0}$. For $t \leq T$ we have

$$
\mathbb{E}\left[|X(t ; 0,1)|^{p}\right] \leq \sup _{t \in[0, T]} \mathbb{E}\left(|X(t ; 0,1)|^{p}\right) .
$$

Now define

$$
C=\max \left(C^{\prime}, \frac{\sup _{t \in[0, T]} \mathbb{E}\left(|X(t ; 0,1)|^{p}\right)}{\min \left(1,(1+T)^{\alpha}\right)}\right) .
$$

Again, we see that $C$ is independent of $X_{0}$. Then for $t \leq T$ we have $\mathbb{E}\left[\left|X\left(t ; 0, X_{0}\right)\right|^{p}\right]=$ $\mathbb{E}\left[\left|X_{0}\right|^{p}\right] \mathbb{E}\left(|X(t ; 0,1)|^{p}\right)$ and so

$$
\begin{aligned}
\mathbb{E}\left[\left|X\left(t ; 0, X_{0}\right)\right|^{p}\right] & \leq \mathbb{E}\left[\left|X_{0}\right|^{p}\right](1+t)^{\alpha} \cdot \frac{\sup _{t \in[0, T]} \mathbb{E}\left(|X(t ; 0,1)|^{p}\right)}{(1+t)^{\alpha}} \\
& \leq \mathbb{E}\left[\left|X_{0}\right|^{p}\right](1+t)^{\alpha} \cdot \frac{\sup _{t \in[0, T]} \mathbb{E}\left(|X(t ; 0,1)|^{p}\right)}{\min \left((1+T)^{\alpha}, 1\right)} \\
& \leq C \mathbb{E}\left[\left|X_{0}\right|^{p}\right](1+t)^{\alpha} .
\end{aligned}
$$

On the other hand, for $t \leq T$ we have

$$
\mathbb{E}\left[\left|X\left(t ; 0, X_{0}\right)\right|^{p}\right]=\mathbb{E}\left[\left|X_{0}\right|^{p}\right] \mathbb{E}\left(|X(t ; 0,1)|^{p}\right) \leq C^{\prime} \mathbb{E}\left[\left|X_{0}\right|^{p}\right](1+t)^{\alpha} \leq C \mathbb{E}\left[\left|X_{0}\right|^{p}\right](1+t)^{\alpha} .
$$

Hence the estimate $\mathbb{E}\left[\left|X\left(t ; 0, X_{0}\right)\right|^{p}\right] \leq C \mathbb{E}\left[\left|X_{0}\right|^{p}\right](1+t)^{\alpha}$ holds for all $t \geq 0$, and this legitimises our definition in (3.2a).

We are concerned with the following notions of asymptotic stability.

Definition 3.2. The equilibrium solution of the SDDE (2.1) is said to be

1. globally polynomially stable in $p$-th mean, if (3.2a) holds with $\alpha_{1}<0$;

2. almost surely globally polynomially stable, if (3.2b) holds with $\alpha_{2}<0$. 
Remark 3.3. If, for every $\epsilon>0$, the solution of the $\operatorname{SDDE}$ (2.1) satisfies

$$
\limsup _{t \rightarrow \infty} \frac{\left|X\left(t ; 0, X_{0}\right)\right|}{t^{\alpha_{2}+\epsilon}}=0, \quad \text { almost surely, }
$$

then the solution is almost surely globally polynomially bounded with constant $\alpha_{2}$, i.e. (3.2b) holds. Moreover, (3.4) implies (3.2b). The term global in the above refers to the fact that we do not require a restriction on the initial value.

\subsection{Results for the deterministic pantograph equation}

First, we provide a comparison result for solutions of pantograph equations. We mention that stochastic comparison arguments are used in the study of stochastic delay differential equations with discrete time lag and their Euler-Maruyama approximations [13], and for ItôVolterra equations [19].

Recall that, for a continuous real-valued function $f$ of a real variable, the Dini derivative $D^{+} f$ is defined as

$$
D^{+} f(t)=\limsup _{\delta \downarrow 0} \frac{f(t+\delta)-f(t)}{\delta} .
$$

Lemma 3.4. Let $\bar{b}>0, q \in(0,1)$. Assume $x$ satisfies

$$
x^{\prime}(t)=\bar{a} x(t)+\bar{b} x(q t), \quad t \geq 0,
$$

where $x(0)>0$ and suppose $t \mapsto p(t)$ is a continuous non-negative function defined on $\mathbb{R}^{+}$satisfying

$$
D^{+} p(t) \leq \bar{a} p(t)+\bar{b} p(q t), \quad t \geq 0
$$

with $0<p(0)<x(0)$. Then $p(t) \leq x(t)$ for all $t \geq 0$.

Proof. See the Appendix.

The following result concerning asymptotic properties of the deterministic pantograph equation has been proved in [29]. The first half of the result (given in [29, Section 4, Theorem 3]) will be of utility in obtaining polynomial upper bounds on the $p^{\text {th }}$-mean of the process; and those bounds are in turn used to obtain estimates on the almost sure asymptotic behaviour of the solution of (2.1). The second part will be employed to establish exponential upper bounds on the solutions of (2.1); it can be found in [29, Section 5, Theorem 5]. The case when $\bar{a}=0$ is covered in [29, Section 6 , Theorem 7]; we have slightly modified notation for convenience. We recall for the third part that for $z \in \mathbb{C}$ that the principal value of the logarithm is defined by

$$
\log (z):=\log |z|+i \operatorname{Arg}(z)
$$

where $\operatorname{Arg}(z) \in(-\pi, \pi]$ is the principal value of the argument of $z$. In particular, for $x<0$, $\log (x)=\log |x|+i \pi$.

Lemma 3.5. Let $x$ be the solution of (2.2).

(i) If $\bar{a}<0$, there exists $C_{1}>0$ such that

$$
\limsup _{t \rightarrow \infty} \frac{|x(t)|}{t^{\gamma}}=C_{1}|x(0)|
$$


where $\gamma \in \mathbb{R}$ obeys

$$
0=\bar{a}+|\bar{b}| q^{\gamma}
$$

Therefore, for some $C>0$, we have

$$
|x(t)| \leq C|x(0)|(1+t)^{\gamma}, \quad t \geq 0 .
$$

(ii) If $\bar{a}>0$, there exists $C>0$ such that

$$
|x(t)| \leq C|x(0)| e^{\bar{a} t}, \quad t \geq 0 .
$$

(iii) If $\bar{a}=0$, define $c^{\prime}=\log (1 / q)>0$ and set

$$
\psi(t):=t^{k}(\log (t))^{h} \exp \left(\frac{1}{2 c^{\prime}}\{\log (t)-\log \log (t)\}^{2}\right),
$$

where

$$
k=\frac{1}{2}+\frac{1}{c^{\prime}}+\frac{1}{c^{\prime}} \log \left(\bar{b} c^{\prime}\right), \quad h=-1-\frac{1}{c^{\prime}} \log \left(\bar{b} c^{\prime}\right) .
$$

Then $x(t)=\mathcal{O}(\psi(t))$ as $t \rightarrow \infty$, and if $x(0) \neq 0$, then $x(t)$ is not $o(\psi(t))$ as $t \rightarrow \infty$.

We can consider $x(0) \neq 0$ in Lemma 3.5, because if $x(0)=0$, then $x(t)=0$ for all $t \geq 0$ and all estimates follow trivially.

The constants $C$ and $C_{1}$ in Lemma 3.5 are independent of $x(0)$, and indeed the estimates (3.7), (3.8) and (3.9) hold for all $t \geq 0$, rather than merely for sufficiently large $t$, as might readily be supposed. This is because the function $x_{1}$, which is the unique continuous solution of (2.2) with initial condition $x_{1}(0)=1$, can be used to express the solution of (2.2) with general initial condition $x(0) \neq 0$. Indeed, we have that $x(t)=x(0) x_{1}(t)$ for all $t \geq 0$. Therefore, applying part (i) of Lemma 3.5 to $x_{1}$, we see that there exists a constant $C_{1}=$ $C_{1}(\bar{a}, \bar{b}, q)>0$

$$
\limsup _{t \rightarrow \infty} \frac{\left|x_{1}(t)\right|}{t^{\gamma}}=C_{1}
$$

where $\gamma$ obeys (3.7). Therefore, there must also exist a constant $C=C(\bar{a}, \bar{b}, q)$ such that $\left|x_{1}(t)\right| \leq C_{1}(1+t)^{\gamma}$ for $t>0$ from which (3.8) immediately follows. An analogous argument applies to part (ii).

We do not discuss in this paper the situation when $\bar{a}=0$ which is covered by part (iii). It can be seen from part (iii) of Lemma 3.5 that

$$
\log |\psi(t)| \sim \frac{1}{2} \frac{1}{\log (1 / q)} \log ^{2}(t), \quad \text { as } t \rightarrow \infty
$$

so therefore as $x=\mathcal{O}(\psi)$ and $x$ is not $o(\psi)$ we have

$$
\limsup _{t \rightarrow \infty} \frac{\log |x(t)|}{\log ^{2}(t)}=\frac{1}{2} \frac{1}{\log (1 / q)} .
$$

Therefore, $x$ enjoys an upper bound which is neither polynomial nor exponential, so we would not expect such bounds to transfer to the corresponding stochastic equation. For this reason such problems are beyond the immediate scope of the paper. In the case $a=0, b>0$, $\rho=0$, however, we can use the methods herein to show that $|X(t)|>0$ for all $t \geq 0$, and that $m(t)=\mathbb{E}[|X(t)|] / \mathbb{E}\left[\left|X_{0}\right|\right.$ solves the differential equation $m^{\prime}(t)=b m(q t)$ for $t \geq 0$ with 
$m(0)=1$. Then the estimate in part (iii) of Lemma 3.5 can be applied to $m$ and thence to $t \mapsto \mathbb{E}[|X(t)|]$. The pure delay stochastic pantograph equation (i.e., with $a=\sigma=0$ )

$$
d X(t)=b X(q t) d t+\rho X(q t) d B(t)
$$

is more likely to inherit the type of asymptotic behaviour in part (iii) of Lemma 3.5, but such a conjecture is the subject of further investigation.

Now, taking as given that the case $\bar{a}=0$ is excluded henceforth from our discussions, we may combine the results of the previous two lemmas to obtain the following explicit estimates on the asymptotic behaviour of continuous functions obeying inequality (3.6).

Lemma 3.6. Suppose $p(0)>0$ and $t \mapsto p(t)$ is a continuous non-negative function defined on $\mathbb{R}^{+}$ satisfying (3.6) where $\bar{b}>0$ and $q \in(0,1)$.

(i) If $\bar{a}<0$, there exists $C>0$ such that

$$
p(t) \leq C p(0)(1+t)^{\gamma}, \quad t \geq 0
$$

where $\gamma$ obeys (3.7).

(ii) If $\bar{a}>0$, there exists $C>0$ such that

$$
p(t) \leq C p(0) e^{\bar{a} t}, \quad t \geq 0 .
$$

Proof. We establish part (i) only; the proof of part (ii) follows similarly. Let $\varepsilon>0$ and $x$ be a solution of (2.2) with $x(0)=(1+\varepsilon) p(0)>p(0)$. By Lemma 3.4, $p(t) \leq x(t)$. By Lemma 3.5 (i), there is $C^{\prime}>0$ such that $x(t) \leq C^{\prime} x(0) t^{\gamma}$, for $t \geq 0$ where $\gamma$ is given by (3.7). But then

$$
p(t) \leq x(t) \leq C^{\prime} x(0) t^{\gamma}=C p(0)(1+t)^{\gamma}
$$

where $C=C^{\prime}(1+\varepsilon)$.

\section{Polynomial asymptotic behaviour}

In this section, we concentrate on giving sufficient conditions under which the asymptotics of the process satisfying (2.1) are polynomially bounded or stable, in both a $p$-th mean $(p=$ $1,2)$ and almost sure sense. The proofs of the $p$-th mean polynomial asymptotic behaviour follow essentially by taking expectations across the process $|X(t)|^{p}$, whose semimartingale decomposition is known by Itô's rule. This yields a functional differential inequality whose solution can be majorised by the solution of a deterministic pantograph equation.

The determination of almost sure polynomial boundedness or stability of the solution of the stochastic pantograph equation uses the ideas of Theorem 4.3.1 in Mao [42]. There the idea is to determine a.s. exponential asymptotic stability of solutions of stochastic functional differential equations with bounded delay, once a decreasing exponential upper bound is obtained for a $p$-th moment. These ideas have been modified to obtain results on almost sure asymptotic stability of Itô-Volterra equations, using the $p$-th mean integrability of the solution [1]. 


\subsection{Polynomial growth bounds in the first and second mean}

In Theorem 4.1 we give sufficient conditions on the parameters $a, b, \sigma$ and $\rho$, such that solutions of (2.1) are polynomially bounded in the first mean and in mean-square. We treat the cases $\rho=0$ and $\rho \neq 0$ separately, because in the first case we can obtain a larger set of parameter values for which the $p$-th moment of the solution is bounded by a polynomial, for $p \leq 1$. Another benefit of a separate analysis for the first mean in the case $\rho=0$, is that we obtain a larger parameter region for which the process is a.s. polynomial bounded, and a.s. polynomial stable, than if we initially obtain a bound on the second mean.

We consider polynomial stability of the solutions of (2.1) in Theorem 4.3.

Theorem 4.1. Let $(X(t))_{t \geq 0}$ be the unique process satisfying (2.1).

(i) Let $\rho=0$, and $\mathbb{E}\left[\left|X_{0}\right|^{2}\right]<\infty$. If $a<0$, there exists a real constant $\alpha$, and a positive constant $C$ such that

$$
\mathbb{E}(|X(t)|) \leq C \mathbb{E}\left(\left|X_{0}\right|\right)(1+t)^{\alpha} \quad \text { for } \quad t \geq 0,
$$

where $\alpha$ is given by

$$
\alpha=\frac{1}{\log q} \log \left(\frac{-a}{|b|}\right)
$$

(ii) Let $\rho \neq 0$, and $\mathbb{E}\left[\left|X_{0}\right|^{4}\right]<\infty$. If $2 a+\sigma^{2}<0$, there exists a real constant $\alpha$, and a positive constant $C$ such that

$$
\mathbb{E}\left(|X(t)|^{2}\right) \leq C \mathbb{E}\left(\left|X_{0}\right|^{2}\right)(1+t)^{\alpha} \quad \text { for } \quad t \geq 0,
$$

where $\alpha$ is given by

$$
\alpha=\frac{2}{\log q} \log \left(\frac{1}{\rho^{2}}\left(\sqrt{|b+\sigma \rho|^{2}-\rho^{2}\left(2 a+\sigma^{2}\right)}-|b+\sigma \rho|\right)\right) .
$$

Remark 4.2. In Theorem 4.1, Part (i), the exponent $\alpha$ is sharp for $b>0$, as

$$
\lim _{t \rightarrow \infty} \frac{\mathbb{E}(|X(t)|)}{t^{\alpha}}=C \mathbb{E}\left(\left|X_{0}\right|\right)
$$

Proof of Remark 4.2. To see this, recall that $X\left(t ; 0, X_{0}\right)=X(t ; 0,1) X_{0}$ for $t \geq 0$. Define $Y(t)=$ $X(t ; 0,1)$ for $t \geq 0$. Then $b>0$ and $\rho=0, Y$ obeys

$$
d Y(t)=(a Y(t)+b Y(q t)) d t+\sigma Y(t) d B(t), \quad t \geq 0 ; \quad Y(0)=1 .
$$

Then it can be shown, because $Y(0)>0$, that $Y(t)>0$ for all $t \geq 0$ almost surely. This is done by defining $\phi(t)=e^{\left(a-\sigma^{2} / 2\right) t+\sigma B(t)}$ for $t \geq 0$. Then $\phi$ obeys the stochastic differential equation

$$
d \phi(t)=a \phi(t) d t+\sigma \phi(t) d B(t), \quad t \geq 0 ; \quad \phi(0)=1 .
$$

Then, using stochastic integration by parts, we deduce that $Q(t):=Y(t) / \phi(t)$ obeys

$$
\begin{aligned}
d Q(t) & =d(Y(t) / \phi(t))=\frac{1}{\phi(t)} d Y(t)+Y(t) d\left(\phi(t)^{-1}\right)-\sigma^{2} Y(t) \phi(t) d t \\
& =b \frac{1}{\phi(t)} Y(q t) d t=b \frac{1}{\phi(t)} \phi(q t) Q(q t) d t .
\end{aligned}
$$


Hence with $\mu(t):=b \phi(q t) / \phi(t)$ for $t \geq 0$, we see that $Q$ obeys the functional differential equation

$$
Q^{\prime}(t)=\mu(t) Q(q t), \quad t \geq 0 ; \quad Q(0)=1
$$

Since $\phi(t)>0$ for all $t \geq 0$, it follows that $\mu(t)>0$ for all $t \geq 0$. Since $Q(0)=1$, we see that $Q(t)>0$ for all $t \geq 0$, using a standard deterministic argument, it follows that $Y(t)=Q(t) \phi(t)>0$ for all $t \geq 0$, as claimed.

Next, by taking expectations across (4.3) leads to

$$
m(t):=\mathbb{E}[Y(t)]=m(0)+\int_{0}^{t}(a m(s)+b m(q s)) d s, \quad t \geq 0 .
$$

Since $m$ is continuous, we get

$$
m^{\prime}(t)=a m(t)+b m(q t), \quad t \geq 0 ; \quad m(0)=1 .
$$

Since $a<0$ and $b>0$, it follows from part (i) of Lemma 3.5 that

$$
\lim _{t \rightarrow \infty} \frac{m(t)}{t^{\alpha}}=C_{1}
$$

where $\alpha$ obeys (4.1). Finally, because $Y(t)>0$ for all $t \geq 0$, and $Y$ and $X_{0}$ are independent we have

$$
m(t) \mathbb{E}\left[\left|X_{0}\right|\right]=\mathbb{E}[Y(t)] \mathbb{E}\left[\left|X_{0}\right|\right]=\mathbb{E}[|Y(t)|] \mathbb{E}\left[\left|X_{0}\right|\right]=\mathbb{E}\left[\left|Y(t) X_{0}\right|\right]=\mathbb{E}\left[\left|X\left(t ; 0, X_{0}\right)\right|\right] .
$$

Therefore

$$
\lim _{t \rightarrow \infty} \frac{\mathbb{E}\left[\left|X\left(t ; 0, X_{0}\right)\right|\right]}{t^{\alpha}}=\lim _{t \rightarrow \infty} \frac{m(t) \mathbb{E}\left[\left|X_{0}\right|\right]}{t^{\alpha}}=C_{1} \mathbb{E}\left[\left|X_{0}\right|\right]
$$

as claimed.

Proof of Theorem 4.1. Part (i): Notice by (2.1) that $X$ is a continuous semimartingale and therefore there exists a semimartingale local time $\Lambda$ for $X$. By the Tanaka-Meyer formula [28, Chap. 3, (7.9)] we therefore have

$$
\begin{aligned}
|X(t)|= & |X(0)|+\int_{0}^{t} \operatorname{sgn}(X(s))(a X(s)+b X(q s)) d s \\
& +\int_{0}^{t} \operatorname{sgn}(X(s)) \sigma X(s) d B(s)+2 \Lambda_{t}(0), \quad \text { a.s. }
\end{aligned}
$$

where $\Lambda_{t}(0)$ is the local time of $X$ at the origin. In fact by Lemma 8.2 and the remark following it, we have

$$
\Lambda_{t}(0)=0 \text { for all } t \geq 0, \text { a.s. }
$$

Thus, for any $t \geq 0, t+h \geq 0,(4.4)$ gives

$$
\begin{aligned}
|X(t+h)|-|X(t)| & =\int_{t}^{t+h}(a|X(s)|+\operatorname{sgn}(X(s)) b X(q s)) d s+\int_{t}^{t+h} \sigma|X(s)| d B(s) \\
& \leq \int_{t}^{t+h}\{a|X(s)|+|b||X(q s)|\} d s+\int_{t}^{t+h} \sigma|X(s)| d B(s) .
\end{aligned}
$$

By Lemma 8.4 (ii), we get, with $m(t)=\mathbb{E}(|X(t)|)$

$$
m(t+h)-m(t) \leq \int_{t}^{t+h}\{a m(s)+|b| m(q s)\} d s .
$$


By Lemma $8.4(\mathrm{i}), t \mapsto m(t)$ is continuous, so

$$
D^{+} m(t) \leq a m(t)+|b| m(q t) .
$$

Note that $m(0)=\mathbb{E}(|X(0)|) \leq\left(\mathbb{E}\left(|X(0)|^{2}\right)\right)^{1 / 2}<\infty$. Since $\alpha$ defined by (4.1) satisfies $a+$ $|b| q^{\alpha}=0$, Lemma 3.6 implies there exists $C>0$ such that

$$
\mathbb{E}(|X(t)|)=m(t) \leq C m(0)(1+t)^{\alpha}=C \mathbb{E}(|X(0)|)(1+t)^{\alpha}, \quad t \geq 0,
$$

as required.

As for the proof of part (ii), in which $\rho \neq 0$, we observe that if $X(t)$ is governed by (2.1), then $Y(t)=X^{2}(t)$ is an Itô process, with semimartingale decomposition given by Itô's rule applied to $X^{2}(t)$ of:

$$
\begin{aligned}
Y(t)=Y(0)+\int_{0}^{t}\{2 a Y(s)+2 b X(s) X(q s)+(\sigma X(s)+ & \left.\rho X(q s))^{2}\right\} d s \\
& +\int_{0}^{t}\{2 \sigma Y(s)+2 \rho X(s) X(q s)\} d B(s),
\end{aligned}
$$

so for $t \geq 0, t+h \geq 0$, we have

$$
\begin{aligned}
Y(t+h)-Y(t)=\int_{t}^{t+h}\{2 a Y(s)+2 b X(s) X(q s) & \left.+(\sigma X(s)+\rho X(q s))^{2}\right\} d s \\
& +\int_{t}^{t+h}\{2 \sigma Y(s)+2 \rho X(s) X(q s)\} d B(s) .
\end{aligned}
$$

Noting that

$$
\begin{aligned}
(2 \sigma Y(s)+2 \rho X(s) X(q s))^{2} & \leq 2\left(4 \sigma^{2} Y^{2}(s)+4 \rho^{2} Y(s) Y(q s)\right) \\
& \leq 8 \sigma X^{4}(s)+4 \rho^{2}\left(X^{4}(s)+X^{4}(q s)\right),
\end{aligned}
$$

and using the fact (Lemma 8.4 (ii)) that

$$
\int_{0}^{t} \mathbb{E}\left(|X(s)|^{4}\right) d s<\infty, \quad \text { for all } \quad t \geq 0
$$

we have

$$
\mathbb{E} \int_{t}^{t+h}(2 \sigma Y(s)+2 \rho X(s) X(q s))^{2} d B(s)=0 .
$$

Let $\eta$ be any positive constant. Using Young's inequality in the form $2 x y \leq \eta^{2} x^{2}+\eta^{-2} y^{2}$ on the right hand side of (4.7) yields

$$
\begin{aligned}
Y(t+h)-Y(t) \leq & \int_{t}^{t+h}\left\{\left(2 a+\sigma^{2}\right) Y(s)+\rho^{2} Y(q s)+|b+\sigma \rho|\left(\eta^{2} Y(s)+\frac{1}{\eta^{2}} Y(q s)\right)\right\} d s \\
& +\int_{t}^{t+h}\{2 \sigma Y(s)+2 \rho X(s) X(q s)\} d B(s) .
\end{aligned}
$$

Let $\widetilde{m}(t)=\mathbb{E}(Y(t))$. Note from Lemma 8.4 (i) that $t \mapsto \widetilde{m}(t)$ is a continuous and (by construction) nonnegative function. Taking expectations both sides of the last inequality, and using (4.8), we therefore get

$$
\begin{aligned}
\widetilde{m}(t+h)-\widetilde{m}(t) \leq & \int_{t}^{t+h}\left(2 a+\sigma^{2}+\eta^{2}|b+\sigma \rho|\right) \widetilde{m}(s) d s \\
& +\int_{t}^{t+h}\left(\rho^{2}+\frac{1}{\eta^{2}}|b+\sigma \rho|\right) \widetilde{m}(q s) d s
\end{aligned}
$$


Using the continuity of $\widetilde{m}$, we now get

$$
D^{+} \widetilde{m}(t) \leq\left(2 a+\sigma^{2}+\eta^{2}|b+\sigma \rho|\right) \widetilde{m}(t)+\left(\frac{1}{\eta^{2}}|b+\sigma \rho|+\rho^{2}\right) \widetilde{m}(q t) .
$$

Since $2 a+\sigma^{2}<0$, we can choose $\eta>0$ such that $\bar{a}=2 a+\sigma^{2}+|b+\sigma \rho| \eta^{2}<0$. Now, using part (i) of Lemma 3.6, there exists $C>0$ and $\alpha \in \mathbb{R}$ such that

$$
\mathbb{E}\left(|X(t)|^{2}\right)=\widetilde{m}(t) \leq C \widetilde{m}(0)(1+t)^{\alpha}=C \mathbb{E}\left(|X(0)|^{2}\right)(1+t)^{\alpha}, \quad t \geq 0 .
$$

Finally we choose $\eta>0$ optimally; i.e., in such a way that $\alpha=\alpha(\eta)$ given by

$$
2 a+\sigma^{2}+\eta^{2}|b+\sigma \rho|+\left(\rho^{2}+\frac{1}{\eta^{2}}|b+\sigma \rho|\right) q^{\alpha}=0
$$

is minimised (such a minimum exists, once $2 a+\sigma^{2}<0$ ). The optimal choice of $\eta$ gives rise to the value of $\alpha$ given in (4.2). This value of $\alpha$ gives the sharpest bound on the growth rate of $\mathbb{E}\left(|X(t)|^{2}\right)$, in the sense that any other choice of $\eta$ gives rise to a larger $\alpha$ than that quoted in (4.2). The proof is thus complete.

The line of reasoning used in Theorem 4.1, taken in conjunction with Lemma 3.6, enables us to obtain polynomial stability of the equilibrium solution of (2.1) in the first and second mean in the sense of Definition 3.2 in certain parameter regions.

Theorem 4.3. Let $(X(t))_{t \geq 0}$ be the process uniquely defined by (2.1).

(i) Let $\rho=0$, and $\mathbb{E}\left[\left|X_{0}\right|^{2}\right]<\infty$. If $a+|b|<0$, there exists $C>0$ and $\alpha<0$ such that

$$
\mathbb{E}(|X(t)|) \leq C \mathbb{E}\left(\left|X_{0}\right|\right)(1+t)^{\alpha}, \quad t \geq 0,
$$

with $\alpha$ given by (4.1).

(ii) Let $\rho \neq 0$, and $\mathbb{E}\left[\left|X_{0}\right|^{4}\right]<\infty$. If $2 a+\sigma^{2}+\rho^{2}+2|b+\sigma \rho|<0$, there exists $C>0, \alpha<0$ such that

$$
\mathbb{E}\left(|X(t)|^{2}\right) \leq C \mathbb{E}\left(\left|X_{0}\right|^{2}\right)(1+t)^{\alpha}, \quad t \geq 0,
$$

with $\alpha$ given by (4.2).

Proof. For part (i), we may use the analysis of Theorem 4.1; we have for some $C>0$

$$
\mathbb{E}(|X(t)|) \leq C \mathbb{E}\left(\left|X_{0}\right|\right)(1+t)^{\alpha},
$$

where $\alpha$ satisfies $a+|b| q^{\alpha}=0$. If $a+|b|<0$, then we must have $\alpha<0$, proving the result.

For part (ii), we revisit the method of proof of Theorem 4.1, part (ii). If $2 a+\sigma^{2}+\rho^{2}+$ $2|b+\sigma \rho|<0$, there exists $\eta>0$ such that

$$
2 a+\sigma^{2}+\eta^{2}|b+\sigma \rho|+\frac{1}{\eta^{2}}|b+\sigma \rho|+\rho^{2}<0 .
$$

In this case, the optimal choice of $\eta$ which minimises $\alpha=\alpha(\eta)$ given in (4.10), yields a constant $\alpha<0$ given by (4.2).

Remark 4.4. In the case $\rho=0$, the solution of (2.1) is asymptotically stable in first mean when the corresponding deterministic problem is asymptotically stable. When $\rho \neq 0$, observe that $2(a+|b|)+(|\sigma|+|\rho|)^{2}<0$ implies $2 a+\sigma^{2}+\rho^{2}+2|b+\sigma \rho|<0$. Therefore, the solution of (2.1) is asymptotically stable in mean square whenever the deterministic problem is asymptotically stable, provided the noise coefficients $\sigma$ and $\rho$ are not too large. 


\subsection{Almost sure polynomial growth bounds}

We can use the estimates of the previous section to determine the polynomial asymptotic behaviour of the solution of (2.1) in an almost sure sense. In Theorem 4.5, we concentrate on the a.s. polynomial boundedness, while Theorem 4.6 concerns the a.s. polynomial stability of solutions of (2.1). Again we treat in both propositions the cases $\rho=0$ and $\rho \neq 0$ separately, obtaining sharper results in the former case.

We note by the Burkholder-Davis-Gundy inequality that there exists a positive, universal, constant $c_{2}$ (universal in the sense that it is independent of the process $X$ and times $t_{1}, t_{2}$ ) such that

$$
\mathbb{E}\left[\sup _{t_{1} \leq s \leq t_{2}}\left(\int_{t_{1}}^{s} X(u) d B(u)\right)^{2}\right] \leq c_{2} \mathbb{E}\left[\int_{t_{1}}^{t_{2}} X^{2}(s) d s\right] .
$$

This bound is of great utility in obtaining estimates on the expected value of the suprema of the process.

We now proceed with the main results of this section.

Theorem 4.5. Let $(X(t))_{t \geq 0}$ be the process uniquely defined by (2.1).

(i) Let $\rho=0, \mathbb{E}\left(\left|X_{0}\right|^{2}\right)<\infty$. If $a<0$, then

$$
\limsup _{t \rightarrow \infty} \frac{\log |X(t)|}{\log t} \leq \alpha+1, \quad \text { a.s., }
$$

where $\alpha$ is defined by (4.1).

(ii) Let $\rho \neq 0, \mathbb{E}\left(\left|X_{0}\right|^{4}\right)<\infty$. If $2 a+\sigma^{2}<0$, then

$$
\limsup _{t \rightarrow \infty} \frac{\log |X(t)|}{\log t} \leq \frac{1}{2}(\alpha+1), \quad \text { a.s., }
$$

where $\alpha$ is defined by (4.2).

Proof. In the proof of part (i) of Theorem 4.1, we established the existence of $C>0$ and $\alpha$ (determined by (4.1)), so that for $t \geq 0$ we have

$$
\mathbb{E}(|X(t)|) \leq C \mathbb{E}\left(\left|X_{0}\right|\right)(1+t)^{\alpha},
$$

whenever $a<0$. Therefore for $t \geq 1$ we have with $C^{*}=C \max \left(1,2^{\alpha}\right) \mathbb{E}\left(\left|X_{0}\right|\right)$

$$
\mathbb{E}(|X(t)|) \leq C \mathbb{E}\left(\left|X_{0}\right|\right) t^{\alpha} \cdot(1+1 / t)^{\alpha} \leq C^{*} t^{\alpha} .
$$

Take $\varepsilon>0$ to be fixed, and define $\lambda=-(\alpha+1+\varepsilon)$. Set $a_{n}=n \tau$, where $\tau^{\frac{1}{2}} c_{2}|\sigma|=\frac{1}{2}$, and $c_{2}$ is the constant in (4.11), so that

$$
c_{2}\left(a_{n+1}-a_{n}\right)^{\frac{1}{2}}|\sigma|=\frac{1}{2}
$$

Moreover, we have

$$
\int_{a_{n}}^{a_{n+1}} s^{\alpha} d s \leq \tau(\tau n)^{\alpha} \max \left(1,2^{\alpha}\right)
$$

To see this, note for $n \geq 1$ that

$$
\frac{1}{a_{n+1}-a_{n}} \int_{a_{n}}^{a_{n+1}}\left(\frac{s}{a_{n}}\right)^{\alpha} d s \leq \max _{a_{n} \leq s \leq a_{n+1}}\left(\frac{s}{a_{n}}\right)^{\alpha} .
$$


The right hand side of this expression equals 1 if $\alpha \leq 0$, while for $\alpha>0$, it is $\left(a_{n+1} / a_{n}\right)^{\alpha}$. However, $\left(a_{n+1} / a_{n}\right)^{\alpha} \leq 2^{\alpha}$, for $\alpha>0$ and $n \geq 1$.

For every $t \in \mathbb{R}^{+}$, there exists $n \in \mathbb{N}$ such that $a_{n} \leq t<a_{n+1}$. Consider $t \in\left[a_{n}, a_{n+1}\right)$, so

$$
X(t)=X\left(a_{n}\right)+\int_{a_{n}}^{t}\{a X(s)+b X(q s)\} d s+\int_{a_{n}}^{t} \sigma X(s) d B(s) .
$$

Using the triangle inequality, taking suprema and expectations over $\left[a_{n}, a_{n+1}\right]$, and noting that $X(t)$ is continuous, we arrive at

$$
\begin{aligned}
\mathbb{E}\left(\sup _{a_{n} \leq t \leq a_{n+1}}|X(t)|\right) \leq \mathbb{E}\left(\left|X\left(a_{n}\right)\right|\right)+\int_{a_{n}}^{a_{n+1}}\{|a| \mathbb{E}(|X(s)|)+|b| & \mathbb{E}(|X(q s)|)\} d s \\
& +\mathbb{E}\left(\sup _{a_{n} \leq t \leq a_{n+1}}\left|\int_{a_{n}}^{t} \sigma X(s) d B(s)\right|\right) .
\end{aligned}
$$

Using (4.11) and (4.13), we can bound the last term on the right hand side of (4.15) for $n \geq 1$ by

$$
\begin{aligned}
& \mathbb{E}\left(\sup _{a_{n} \leq t \leq a_{n+1}}\left|\int_{a_{n}}^{t} \sigma X(s) d B(s)\right|\right) \\
& \leq c_{2} \mathbb{E}\left(\int_{a_{n}}^{a_{n+1}} \sigma^{2} X^{2}(s) d s\right)^{\frac{1}{2}} \leq c_{2} \mathbb{E}\left(\left(a_{n+1}-a_{n}\right) \sigma^{2} \sup _{a_{n} \leq t \leq a_{n+1}} X^{2}(t)\right)^{\frac{1}{2}} \\
& \quad=\frac{1}{2} \mathbb{E}\left(\sup _{a_{n} \leq t \leq a_{n+1}}|X(t)|\right) .
\end{aligned}
$$

Hence, for $n$ sufficiently large, from (4.12), (4.14), (4.15) and (4.16) we obtain

$$
\begin{aligned}
\mathbb{E}\left(\sup _{a_{n} \leq t \leq a_{n+1}} \mid X(t)\right) & \leq 2 \mathbb{E}\left(\left|X\left(a_{n}\right)\right|\right)+2 \int_{a_{n}}^{a_{n+1}}\{|a| \mathbb{E}(|X(s)|)+|b| \mathbb{E}(|X(q s)|)\} d s \\
& \leq 2 C^{*} a_{n}^{\alpha}+2\left(|a| C^{*}+|b| C^{*} q^{\alpha}\right) \int_{a_{n}}^{a_{n+1}} s^{\alpha} d s \leq \tilde{C} n^{\mu \alpha}
\end{aligned}
$$

where $\tilde{C}=2 C^{*} \tau^{\alpha}+2 C^{*} \tau^{\alpha+1}\left(|a|+|b| q^{\alpha}\right) \max \left(1,2^{\alpha}\right)$. Thus, by Markov's inequality, for every $\gamma>0$, we have

$$
\begin{aligned}
\mathbb{P}\left(\sup _{a_{n} \leq t \leq a_{n+1}}|X(t)| n^{-\alpha-1-\varepsilon} \geq \gamma\right) & \leq \frac{1}{\gamma} \mathbb{E}\left(\sup _{a_{n} \leq t \leq a_{n+1}}|X(t)| n^{-\alpha-1-\varepsilon}\right) \\
& \leq \frac{1}{\gamma} \frac{1}{n^{1+\varepsilon}} \frac{1}{n^{\alpha}} \cdot \tilde{C} n^{\alpha}=\frac{\tilde{C}}{\gamma} \frac{1}{n^{1+\varepsilon}} .
\end{aligned}
$$

Therefore, by the first Borel-Cantelli Lemma, we must have

$$
\lim _{n \rightarrow \infty} \sup _{a_{n} \leq t \leq a_{n+1}}|X(t)| n^{\lambda}=0 \quad \text { a.s. }
$$

For each $t \in \mathbb{R}^{+}$, there exists $n(t) \in \mathbb{N}$ such that $\tau n(t) \leq t<\tau(n(t)+1)$. Therefore,

$$
\lim _{t \rightarrow \infty} \frac{t}{\tau n(t)}=1
$$


Using (4.17) and (4.18), we have

$$
\begin{aligned}
\limsup _{t \rightarrow \infty}|X(t)| t^{\lambda} & \leq \limsup _{t \rightarrow \infty} \sup _{\tau n(t) \leq s \leq \tau(n(t)+1)}|X(s)|(\tau n(t))^{\lambda} \cdot \limsup _{t \rightarrow \infty}\left(\frac{t}{\tau n(t)}\right)^{\lambda} \\
& =\limsup _{n \rightarrow \infty} \sup _{a_{n} \leq s \leq a_{n+1}}|X(s)| n^{\lambda} \cdot \limsup _{t \rightarrow \infty}\left(\frac{t}{\tau n(t)}\right)^{\lambda}=0, \quad \text { a.s. }
\end{aligned}
$$

Therefore

$$
\limsup _{t \rightarrow \infty} \frac{\log |X(t)|}{\log t} \leq-\lambda=\alpha+1+\varepsilon, \quad \text { a.s. }
$$

Letting $\varepsilon \downarrow 0$ through the rationals completes the proof.

The proof of part (ii) is very similar, and the outline only will be sketched here. By hypothesis, Theorem 4.1 tells us that there exists $\alpha$ satisfying (4.10) such that

$$
\mathbb{E}\left(|X(t)|^{2}\right) \leq C \mathbb{E}\left(\left|X_{0}\right|^{2}\right)(1+t)^{\alpha} .
$$

Once again, this means for all $t \geq 1$ we have

$$
\mathbb{E}\left(|X(t)|^{2}\right) \leq C^{*} t^{\alpha} .
$$

Define, for every $\varepsilon>0, \lambda=-(\alpha+1+\varepsilon)$ and $\varepsilon \in(0,1)$ as before. Define $a_{n}$ as above. To get a bound on $\mathbb{E}\left(\sup _{a_{n} \leq t \leq a_{n+1}} X^{2}(t)\right)$, proceed as for equation (4.15) to obtain

$$
\begin{aligned}
\mathbb{E}\left(\sup _{a_{n} \leq t \leq a_{n+1}} X^{2}(t)\right) \leq 3\left\{\mathbb{E}\left(X^{2}\left(a_{n}\right)\right)\right. & +\mathbb{E}\left(\int_{a_{n}}^{a_{n+1}}|a X(s)+b X(q s)| d s\right)^{2} \\
& \left.+\mathbb{E}\left[\sup _{a_{n} \leq t \leq a_{n+1}}\left(\int_{a_{n}}^{t}(\sigma X(s)+\rho X(q s)) d B(s)\right)^{2}\right]\right\} .
\end{aligned}
$$

By (4.14), the second term on the right hand side of (4.19) has as a bound (for $n \geq 1$ ):

$$
\begin{aligned}
\mathbb{E}\left(\int_{a_{n}}^{a_{n+1}}|a X(s)+b X(q s)| d s\right)^{2} & \leq\left(2 a^{2} C^{*}+2 b^{2} C^{*} q^{\alpha}\right)\left(a_{n+1}-a_{n}\right) \int_{a_{n}}^{a_{n+1}} s^{\alpha} d s \\
& \leq\left(2 a^{2} C^{*}+2 b^{2} C^{*} q^{\alpha}\right) \max \left(1,2^{\alpha}\right) \cdot \tau^{\alpha+1} n^{\alpha} .
\end{aligned}
$$

Using the Burkholder-Davis-Gundy inequality and (4.14), the third term on the right hand side of (4.19) has as a bound (for $n \geq 1$ ):

$$
\begin{aligned}
\mathbb{E}\left[\sup _{a_{n} \leq t \leq a_{n+1}}\left(\int_{a_{n}}^{t}(\sigma X(s)+\rho X(q s)) d B(s)\right)^{2}\right] \\
\quad \leq c_{2} \mathbb{E}\left[\int_{a_{n}}^{a_{n+1}}(\sigma X(s)+\rho X(q s))^{2} d s\right] \\
\leq c_{2}\left(2 \sigma^{2} C^{*}+2 \rho^{2} C^{*} q^{\alpha}\right)\left(a_{n+1}-a_{n}\right) \int_{a_{n}}^{a_{n+1}} s^{\alpha} d s \\
\leq c_{2}\left(2 \sigma^{2} C^{*}+2 \rho^{2} C^{*} q^{\alpha}\right) \max \left(1,2^{\alpha}\right) \cdot \tau^{\alpha+1} n^{\alpha} .
\end{aligned}
$$

Inserting these estimates into (4.19), we obtain

$$
\mathbb{E}\left(\sup _{a_{n} \leq t \leq a_{n+1}} X^{2}(t)\right) \leq \tilde{C} n^{\alpha},
$$

where $\tilde{C}$ is some $n$-independent constant. The rest of the proof goes through as above. 
The results on polynomial stability in the first and second mean can be used to establish almost sure stability of solutions of (2.1) with a polynomial upper bound on the decay rate.

Theorem 4.6. Let $(X(t))_{t \geq 0}$ be the process uniquely defined by (2.1).

(i) Let $\rho=0, \mathbb{E}\left(\left|X_{0}\right|^{2}\right)<\infty$. If $a+|b| / q<0$, then $\alpha$, defined by (4.1), satisfies $\alpha<-1$, and we have

$$
\limsup _{t \rightarrow \infty} \frac{\log |X(t)|}{\log t} \leq \alpha+1, \quad \text { a.s., }
$$

so $X(t) \rightarrow 0$ as $t \rightarrow \infty$, a.s.

(ii) Let $\rho \neq 0, \mathbb{E}\left(\left|X_{0}\right|^{4}\right)<\infty$. If

$$
2 a+\sigma^{2}+\frac{\rho^{2}}{q}+\frac{2}{\sqrt{q}}|b+\sigma \rho|<0,
$$

then $\alpha$, defined by (4.2), satisfies $\alpha<-1$, and we have

$$
\limsup _{t \rightarrow \infty} \frac{\log |X(t)|}{\log t} \leq \frac{1}{2}(\alpha+1) \text {, a.s., }
$$

so $X(t) \rightarrow 0$ as $t \rightarrow \infty$, a.s.

Proof. In part (i) of the theorem, we need $\alpha$ defined by (4.1) to satisfy $\alpha<-1$. Considering (4.1), we see that $a+|b| / q<0$ implies $\alpha<-1$.

The proof of part (ii) follows along identical lines. Suppose (4.20) holds. If we choose $\eta^{2}=1 / \sqrt{q}$, then

$$
2 a+\sigma^{2}+\eta^{2}|b+\sigma \rho|+\left(\rho^{2}+\frac{1}{\eta^{2}}|b+\sigma \rho|\right) \frac{1}{q}<0,
$$

and so $\alpha$ defined by (4.2) satisfies $\alpha<-1$.

\section{Exponential upper bounds}

In [43], it is shown that stochastic delay differential equations, or stochastic functional differential equations with bounded delay, are a.s. bounded by increasing exponential functions, provided that the coefficients of the equation satisfy global linear bounds. More precisely, Mao shows that the top Lyapunov exponent is bounded almost surely by a finite constant. For the stochastic pantograph equation, we similarly show that all solutions have top a.s. and $p$-th mean $(p=1,2)$ Lyapunov exponents which are bounded by finite constants. We consider only parameter regions in which the polynomial boundedness of the solution of (2.1) has not been established, as the a.s. exponential upper bound (respectively, the $p$-th mean exponential upper bound) is a direct consequence of the a.s. polynomial boundedness (respectively, $p$-th mean polynomial boundedness) of the solution.

Therefore, when $a>0$ and $\rho=0$, we show that there is an exponential upper bound on solutions in a first mean and almost sure sense; when $a<0$ and $\rho=0$, we already know that there is a polynomial bound in first mean and almost surely. When $\rho \neq 0$, and $2 a+\sigma^{2}>0$, we show that there is an exponential upper bound on solutions in a mean square and almost sure sense; when $2 a+\sigma^{2}<0$ and $\rho \neq 0$, we have already established that there is a polynomial mean square and almost sure bound on solutions. However, because 
these are upper bounds, we cannot say whether the only classes of asymptotic behaviour are of polynomial or exponentially growing type. Nonetheless, these are the only classes of behaviour exhibited by deterministic pantograph equations, and, in a later work (where $\rho=0$ ), we establish that solutions are either exponentially growing, or cannot grow (or decay) as fast as any exponential.

In Theorem 5.1 below, we obtain an exponential upper bound on the $p$-th mean $(p=$ $1,2)$ using the comparison principle obtained earlier in this paper, and obtain almost sure exponential upper bounds by using ideas of Theorem 4.3.1 in [42] for stochastic differential equations, which are developed in Theorem 5.6.2 in [43] for stochastic equations with delay.

Theorem 5.1. Let $(X(t))_{t \geq 0}$ be the process satisfying (2.1).

(i) Let $\rho=0, \mathbb{E}\left(\left|X_{0}\right|^{2}\right)<\infty$. If $a>0$, then

$$
\mathbb{E}(|X(t)|) \leq C \mathbb{E}\left(\left|X_{0}\right|\right) e^{a t},
$$

and

$$
\limsup _{t \rightarrow \infty} \frac{1}{t} \log |X(t)| \leq a \quad \text { a.s. }
$$

(ii) Let $\rho \neq 0, \mathbb{E}\left[\left|X_{0}\right|^{2}\right]<\infty$. If $2 a+\sigma^{2}>0$, then

$$
\mathbb{E}\left(|X(t)|^{2}\right) \leq C \mathbb{E}\left(\left|X_{0}\right|^{2}\right) e^{\left(2 a+\sigma^{2}\right) t},
$$

and

$$
\limsup _{t \rightarrow \infty} \frac{1}{t} \log |X(t)| \leq a+\frac{1}{2} \sigma^{2} \quad \text { a.s. }
$$

Proof. The bounds on expectations follow from Lemma 3.6 part (ii). For part (i) (where $\rho=0$ ) the proof of equation (4.6) in Theorem 4.1 part (i), together with part (ii) of Lemma 3.6 gives

$$
\mathbb{E}(|X(t)|)=m(t) \leq C m(0) e^{a t}=C \mathbb{E}\left(\left|X_{0}\right|\right) e^{a t}
$$

for some $C>0$.

For part (ii) (where $\rho \neq 0$ ) the proof of (4.9) in Theorem 4.1 part (ii) gives

$$
\mathbb{E}\left(|X(t)|^{2}\right)=\widetilde{m}(t) \leq C \widetilde{m}(0) e^{\bar{a} t}=C \mathbb{E}\left(|X(0)|^{2}\right) e^{\bar{a} t}, \quad t \geq 0
$$

for some $C>0$, where $\bar{a}=2 a+\sigma^{2}+|b+\sigma \rho| \eta^{2}$. As $\eta$ can be chosen arbitrarily small, we have

$$
\mathbb{E}\left(|X(t)|^{2}\right) \leq C(\varepsilon) e^{\left(2 a+\sigma^{2}+\varepsilon\right) t} \mathbb{E}\left(|X(0)|^{2}\right)
$$

for every $\varepsilon>0$. This gives the desired result.

This analysis now enables us to obtain an exponential upper bound on

$$
t \mapsto \mathbb{E}\left(\sup _{0 \leq s \leq t} X^{2}(s)\right) .
$$

The proof follows the idea of the proof of Theorem 4.3.1 in Mao [42], and related results in Mao [43]. It also is similar to earlier results in this paper, so we present an outline for part (ii) only. 
Using the inequality $(a+b+c)^{2} \leq 3\left(a^{2}+b^{2}+c^{2}\right)$, we get

$$
X^{2}(t) \leq 3 X^{2}(0)+3\left(\int_{0}^{t}\{a X(s)+b X(q s)\} d s\right)^{2}+3\left(\int_{0}^{t}\{\sigma X(s)+\rho X(q s)\} d B(s)\right)^{2},
$$

so using the Cauchy-Schwarz inequality, taking suprema, and then expectations on both sides of the inequality, we arrive at

$$
\begin{array}{rl}
\mathbb{E}\left(\sup _{0 \leq t \leq T} X^{2}(t)\right) \leq 3 \mathbb{E}\left(X^{2}(0)\right)+3 T \int_{0}^{T} & \mathbb{E}(|a X(s)+b X(q s)|)^{2} d s \\
& +3 \mathbb{E}\left[\sup _{0 \leq t \leq T}\left(\int_{0}^{t}\{\sigma X(s)+\rho X(q s)\} d B(s)\right)^{2}\right],
\end{array}
$$

for any $T>0$. The second term on the right-hand side can be bounded using the inequality $(a+b)^{2} \leq 2\left(a^{2}+b^{2}\right)$. The third term can be bounded by the Burkholder-Davis-Gundy inequality. Therefore, by (4.11), there exists a $T$-independent constant $c_{2}>0$ such that

$$
\begin{aligned}
\mathbb{E}\left(\sup _{0 \leq t \leq T} X^{2}(t)\right) \leq 3 \mathbb{E}\left(X^{2}(0)\right)+3 T \int_{0}^{T}\left\{2 a^{2}\right. & \left.\mathbb{E}|X(s)|^{2}+2 b^{2} \mathbb{E}|X(q s)|^{2}\right\} d s \\
& +3 c_{2} \int_{0}^{T}\left\{2 \sigma^{2} \mathbb{E}|X(s)|^{2}+2 \rho^{2} \mathbb{E}|X(q s)|^{2}\right\} d s .
\end{aligned}
$$

Noting that $q \in(0,1)$, we now appeal to (5.1) to show for each fixed $\varepsilon>0$ that there exists a $C_{3}(\varepsilon)>0$ such that

$$
\mathbb{E}\left(\sup _{0 \leq t \leq T} X^{2}(t)\right) \leq C_{3}(\varepsilon)(T+1) \exp \left(\left(2|a|+\sigma^{2}+\varepsilon\right) T\right) .
$$

Let $\lambda=|a|+\frac{1}{2} \sigma^{2}$, so that for each fixed $\varepsilon>0$ we have

$$
\mathbb{E}\left[\left(\frac{\sup _{0 \leq s \leq t}|X(s)|}{e^{(\lambda+\varepsilon) t}}\right)^{2}\right] \leq C_{3}(\varepsilon)(t+1) \exp (-\varepsilon t) .
$$

Using Chebyshev's inequality and the first Borel-Cantelli Lemma, we see that for every fixed $\varepsilon \in(0,1)$ there is an almost sure event $\Omega_{\varepsilon}$ such that

$$
\limsup _{t \rightarrow \infty}|X(t, \omega)| e^{-(\lambda+\varepsilon) t} \leq e^{\lambda+1}, \quad \text { for } \omega \in \Omega_{\varepsilon} .
$$

Therefore for $\omega \in \Omega_{\varepsilon}$

$$
\limsup _{t \rightarrow \infty} \frac{1}{t} \log |X(t, \omega)| \leq \lambda+\varepsilon
$$

Let $\Omega^{*}=\cap_{n \in \mathbb{N}} \Omega_{1 / n}$. Then $\mathbb{P}\left[\Omega^{*}\right]=1$ and for all $\omega \in \Omega^{*}$ and all $n \in \mathbb{N}$, we have

$$
\limsup _{t \rightarrow \infty} \frac{1}{t} \log |X(t, \omega)| \leq \lambda+\frac{1}{n} \text {. }
$$

Hence

$$
\limsup _{t \rightarrow \infty} \frac{1}{t} \log |X(t, \omega)| \leq \lambda
$$

for all $\omega \in \Omega^{*}$, as required. 


\section{Generalisations of the scalar stochastic pantograph equation}

In this section, we consider some generalisations of the scalar stochastic pantograph equation, and show, with some restrictions and additional analysis, that our stability and asymptotic analysis extends to cover these more general problems.

In the first subsection, we consider the scalar stochastic pantograph equation with arbitrarily many proportional delays

$$
d X(t)=\left(a X(t)+\sum_{i=1}^{n} b_{i} X\left(q_{i} t\right)\right) d t+\left(\sigma X(t)+\sum_{i=1}^{m} \sigma_{i} X\left(r_{i} t\right)\right) d B(t)
$$

where $q_{i} \in(0,1)$ for $i=1, \ldots, n$ and $r_{i} \in(0,1)$ for $i=1, \ldots, m$ are sets of distinct real numbers, $a, \sigma \in \mathbb{R}, b_{i} \in \mathbb{R}$ for $i=1, \ldots, n$, and $\sigma_{i} \in \mathbb{R}$ for $i=1, \ldots, m$. We assume $(B(t))_{t \geq 0}$ is a standard one-dimensional Brownian motion. The equation (6.1) is a stochastic version of the pantograph equation

$$
x^{\prime}(t)=\operatorname{ax}(t)+\sum_{i=1}^{\infty} b_{i} x\left(q_{i} t\right), \quad t \geq 0
$$

studied by Liu [37]. The asymptotic behaviour of (6.2) as $t \rightarrow \infty$ bears many similarities to that of (2.2), and we will use results about the asymptotic behaviour of (6.2) in order to obtain estimates on the asymptotic behaviour of (6.1).

In the second subsection, we study the finite dimensional stochastic pantograph equation

$$
d X(t)=(A X(t)+B X(q t)) d t+(\Sigma X(t)+\Theta X(q t)) d B(t)
$$

where $A, B, \Sigma, \Theta \in M_{d, d}(\mathbb{R})$, the set of all square $d \times d$ matrices with real entries. To guarantee a unique solution, we must specify $\mathbb{E}\left(\left\|X_{0}\right\|^{2}\right)<\infty$. Here $\|x\|$ denotes the Euclidean norm for $x \in \mathbb{R}^{d}$. We denote by $\langle x, y\rangle$ the standard inner product on for $x, y \in \mathbb{R}^{d}$, and, with a slight abuse of notation, $\|C\|$ for the matrix norm of $C \in M_{d, d}(\mathbb{R})$, where the norm is induced from the standard Euclidean norm. Equation (6.3) is a stochastic complement to the finite dimensional deterministic pantograph equation studied by Carr and Dyson [18].

\subsection{Scalar equation with many delays}

We need some preliminary results in order to obtain asymptotic estimates on (6.1).

Consider the equation

$$
x^{\prime}(t)=\tilde{a} x(t)+\sum_{i=1}^{n}\left|\tilde{b}_{i}\right| x\left(p_{i} t\right),
$$

where $\tilde{a}<0$ and $\tilde{b}_{i} \in \mathbb{R}$. Define the set

$$
\tilde{E}=\left\{z \in \mathbb{C}: \tilde{a}+\sum_{i=1}^{n}\left|\tilde{b}_{i}\right| p_{i}^{z}=0\right\}
$$

and

$$
\tilde{\alpha}=\sup \{\Re e(z): z \in \tilde{E}\} .
$$

Then we obtain the following result. 
Lemma 6.1. Suppose that at least one $\left|\tilde{b}_{i}\right| \neq 0$ in (6.4). Then, there exists $\alpha \in \tilde{E} \cap \mathbb{R}$ such that $\alpha=\tilde{\alpha}$. Moreover, $\alpha<0$ if and only if

$$
\tilde{a}+\sum_{i=1}^{n}\left|\tilde{b}_{i}\right|<0
$$

and $\alpha<-1$ if and only if

$$
\tilde{a}+\sum_{i=1}^{n} \frac{1}{p_{i}}\left|\tilde{b}_{i}\right|<0
$$

Proof. Let $f(z)=a+\sum_{i=1}^{n}\left|\tilde{b}_{i}\right| p_{i}^{z}$. On $\mathbb{R}, f$ is decreasing and continuous, so as $\lim _{z \rightarrow-\infty} f(z)=$ $\infty$, and $\lim _{z \rightarrow \infty} f(z)=a<0, f$ has a unique real zero at $\alpha$. Clearly, $\alpha<0$ if and only if (6.7) holds, and $\alpha<-1$ if and only if (6.8) is true.

To show that $\alpha$ is greater than or equal to the real part of any other member of $\tilde{E}$, consider $z \in \tilde{E}$ with $z=z_{1}+i z_{2}$. Then

$$
\Re e(f(z))=a+\sum_{i=1}^{n}\left|\tilde{b}_{i}\right| p_{i}^{z_{1}} \cos \left(z_{2} \log p_{i}\right) \leq f\left(z_{1}\right) .
$$

If $z_{1}>\alpha$, then $0=\Re e(f(z))=f\left(z_{1}\right)<f(\alpha)=0$, a contradiction. Hence $\tilde{\alpha}=\alpha$.

The relevance of $\tilde{\alpha}$ defined by (6.6) is explained by Theorem 2.14 in [37]. There, it is shown that every solution of (6.4) satisfies

$$
x(t)=\mathcal{O}\left(t^{\beta}\right), \quad \text { as } t \rightarrow \infty
$$

for any $\beta>\tilde{\alpha}$, where $\tilde{\alpha}$ is defined by (6.6). As a consequence of Lemma 6.1 and (6.9), we obtain the following result.

Lemma 6.2. Let $\alpha$ be the real member of $\tilde{E}$ defined by (6.5), and $x$ be any solution of (6.4). Then we have

$$
\limsup _{t \rightarrow \infty} \frac{\log |x(t)|}{\log t} \leq \alpha
$$

We use these results to obtain asymptotic estimates on a parameterised family of equations of the form (6.4).

Towards this end, let $\left(v_{i}\right)_{i=1, \ldots, n},\left(\mu_{i}\right)_{i=1, \ldots, m},\left(\lambda_{i}\right)_{i=1, \ldots, m}$ be as yet unspecified sequences of positive real numbers, and define the equation

$$
\begin{aligned}
y^{\prime}(t)=\left(2 a+\sigma^{2}+\sum_{i=1}^{n}\left|b_{i}\right| v_{i}^{2}+\sum_{j=1}^{m}|\sigma|\left|\sigma_{j}\right| \lambda_{j}^{2}\right) y(t) & +\sum_{i=1}^{n}\left|b_{i}\right| \frac{1}{v_{i}^{2}} y\left(q_{i} t\right) \\
& +\sum_{l=1}^{m} \sigma_{l}^{2} \mu_{l}{ }^{2} \cdot \sum_{j=1}^{m} \mu_{j}^{2} y\left(r_{j} t\right)+\sum_{j=1}^{m}|\sigma|\left|\sigma_{j}\right| \frac{1}{\lambda_{j}^{2}} y\left(r_{j} t\right) .
\end{aligned}
$$

We will write $y$ above as $y_{v, \lambda, \mu}$.

Lemma 6.3. Suppose that $y=y_{v, \lambda, u}$ is a solution of (6.11). Then each of the following is true.

(i) If

$$
2 a+\sigma^{2}<0,
$$

there exists $(\nu, \lambda, \mu)=\left(v^{*}, \lambda^{*}, \mu^{*}\right)$ and $\alpha \in \mathbb{R}$ such that

$$
\limsup _{t \rightarrow \infty} \frac{\log \left|y_{v^{*}, \lambda^{*}, \mu^{*}}(t)\right|}{\log t} \leq \alpha .
$$


(ii) If

$$
2 a+2 \sum_{i=1}^{n}\left|b_{i}\right|+\left(|\sigma|+\sum_{j=1}^{m}\left|\sigma_{j}\right|\right)^{2}<0,
$$

there exists $(\nu, \lambda, \mu)=\left(v^{*}, \lambda^{*}, \mu^{*}\right)$ and $\alpha<0$ such that (6.13) holds.

(iii) If

$$
2 a+2 \sum_{i=1}^{n} \frac{\left|b_{i}\right|}{\sqrt{q_{i}}}+\left(|\sigma|+\sum_{j=1}^{m} \frac{\left|\sigma_{j}\right|}{\sqrt{r_{j}}}\right)^{2}<0,
$$

there exists $(\nu, \lambda, \mu)=\left(v^{*}, \lambda^{*}, \mu^{*}\right)$ and $\alpha<-1$ such that (6.13) holds.

Proof. Notice that (6.11) conforms to the form of (6.4). To establish (i), we see by comparing (6.11) with (6.4) that the conclusion of Lemma 6.2 can be applied to $y=y_{v, \lambda, \mu}$ provided

$$
2 a+\sigma^{2}+\sum_{i=1}^{n}\left|b_{i}\right| v_{i}^{2}+\sum_{j=1}^{m}|\sigma|\left|\sigma_{j}\right| \lambda_{j}^{2}<0
$$

By choosing the $\nu^{\prime}$ s and $\lambda$ 's arbitrarily small, we see that (6.12) suffices to establish (6.16), and thereby (6.13) for $\alpha \in \mathbb{R}$. To prove (ii), by analogy to (6.5) and (6.6), we introduce for $y=y_{v, \lambda, \mu}$ the set

$$
\begin{aligned}
E(\nu, \lambda, \mu)=\left\{z \in \mathbb{C}: 2 a+\sigma^{2}+\sum_{i=1}^{n}\left|b_{i}\right| v_{i}^{2}\right. & +\sum_{j=1}^{m}|\sigma|\left|\sigma_{j}\right| \lambda_{j}{ }^{2}+\sum_{i=1}^{n}\left|b_{i}\right| \frac{1}{v_{i}{ }^{2}} q_{i}{ }^{z} \\
& \left.+\sum_{l=1}^{m} \sigma_{l}{ }^{2} \mu_{l}{ }^{-2} \cdot \sum_{j=1}^{m} \mu_{j}{ }^{2} r_{j}{ }^{z}+\sum_{j=1}^{m}|\sigma|\left|\sigma_{j}\right| \frac{1}{\lambda_{j}{ }^{2}} r_{j}^{z}=0\right\},
\end{aligned}
$$

and let

$$
\alpha(\nu, \lambda, \mu)=\sup \{\Re e(z): z \in E(\nu, \lambda, \mu)\} .
$$

By Lemma 6.1 and (6.7), we see that $\alpha(v, \lambda, v)<0$ if

$$
2 a+\sigma^{2}+\sum_{i=1}^{n}\left|b_{i}\right|\left(v_{i}^{2}+\frac{1}{v_{i}^{2}}\right)+\sum_{j=1}^{m}|\sigma|\left|\sigma_{j}\right|\left(\lambda_{j}^{2}+\frac{1}{\lambda_{j}^{2}}\right)+\sum_{l=1}^{m} \sigma_{l}^{2} \mu_{l}{ }^{2} \cdot \sum_{j=1}^{m} \mu_{j}^{2}<0 .
$$

In particular, if (6.14) is true, we see that by choosing $v_{i}^{*}=1, \lambda_{j}^{*}=1, \mu_{j}^{*}=\sqrt{\left|\sigma_{j}\right|}$ that (6.19) is satisfied, and so by Lemma 6.2, the estimate (6.13) holds with $\alpha=\alpha\left(v^{*}, \lambda^{*}, \mu^{*}\right)<0$.

Part (iii) follows similarly: Lemma 6.1 and (6.8) ensure that $\alpha(v, \lambda, \mu)$ defined by (6.18) satisfies $\alpha(\nu, \lambda, \mu)<-1$ if

$$
2 a+\sigma^{2}+\sum_{i=1}^{n}\left|b_{i}\right|\left(v_{i}^{2}+\frac{1}{q_{i} v_{i}^{2}}\right)+\sum_{j=1}^{m}|\sigma|\left|\sigma_{j}\right|\left(\lambda_{j}^{2}+\frac{1}{r_{j} \lambda_{j}^{2}}\right)+\sum_{l=1}^{m} \sigma_{l}^{2} \mu_{l}{ }^{-2} \cdot \sum_{j=1}^{m} \mu_{j}{ }^{2} \frac{1}{r_{j}}<0 .
$$

In particular, if (6.15) is true, we see that by choosing

$$
\left(v_{i}^{*}\right)^{2}=\frac{1}{\sqrt{q_{i}}}, \quad\left(\lambda_{j}^{*}\right)^{2}=\frac{1}{\sqrt{r_{j}}}, \quad\left(\mu_{j}^{*}\right)^{2}=\frac{\left|\sigma_{j}\right|}{\sqrt{r_{j}}}
$$

the inequality (6.20) is satisfied, and so by Lemma 6.2 the estimate (6.13) stands with $\alpha=$ $\alpha\left(v^{*}, \lambda^{*}, \mu^{*}\right)<-1$. 
The following elementary inequality is also important in our analysis.

Lemma 6.4. Suppose that $\sigma, x \in \mathbb{R}$, and $\left(x_{i}\right)_{i=1, \ldots, m},\left(\sigma_{i}\right)_{i=1, \ldots, m}$ are any two real sequences. If $\left(\lambda_{i}\right)_{i=1, \ldots, m},\left(\mu_{i}\right)_{i=1, \ldots, m}$ are two sequences of positive real numbers, then

$$
\left(\sigma x+\sum_{i=1}^{m} \sigma_{i} x_{i}\right)^{2} \leq \sigma^{2} x^{2}+\sum_{i=1}^{m}|\sigma|\left|\sigma_{i}\right|\left(\lambda_{i}^{2} x^{2}+\frac{1}{\lambda_{i}^{2}} x_{i}^{2}\right)+\sum_{l=1}^{m} \sigma_{l}^{2} \mu_{l}^{-2} \cdot \sum_{j=1}^{m} \mu_{j}^{2} x_{j}^{2} .
$$

Proof. Using the inequality $2 x x_{i} \leq \lambda_{i}^{2} x^{2}+\lambda_{i}^{-2} x_{i}^{2}$, we get

$$
\left(\sigma x+\sum_{i=1}^{m} \sigma_{i} x_{i}\right)^{2} \leq \sigma^{2} x^{2}+\sum_{i=1}^{m}|\sigma| \sigma_{i} \mid\left(\lambda_{i}^{2} x^{2}+\frac{1}{\lambda_{i}^{2}} x_{i}^{2}\right)+\left(\sum_{i=1}^{m} \sigma_{i} x_{i}\right)^{2} .
$$

Applying the Cauchy-Schwarz inequality to the last term gives

$$
\left(\sum_{i=1}^{m} \sigma_{i} x_{i}\right)^{2} \leq \sum_{i=1}^{m} \sigma_{i}^{2} \mu_{i}^{-2} \cdot \sum_{i=1}^{m} \mu_{i}^{2} x_{i}^{2}
$$

proving the result.

With these estimates, we can establish sufficient conditions to ensure the polynomial asymptotic behaviour of solutions of (6.1).

Theorem 6.5. Let $(X(t))_{t \geq 0}$ be the solution of (6.1) with $\mathbb{E}\left(\left|X_{0}\right|^{4}\right)<\infty$. Then the following holds:

(i) Suppose $2 a+\sigma^{2}<0$. Then there exists $C>0$ and $\alpha \in \mathbb{R}$ such that

$$
\limsup _{t \rightarrow \infty} \frac{\log \mathbb{E}\left[|X(t)|^{2}\right]}{\log t} \leq \alpha
$$

and

$$
\limsup _{t \rightarrow \infty} \frac{\log |X(t)|}{\log t} \leq \frac{1}{2}(\alpha+1), \text { a.s. }
$$

Therefore, the process is polynomially bounded in mean-square and almost surely polynomially bounded.

(ii) Suppose (6.14) holds. Then there exists $\alpha<0$ such that (6.21) holds. Therefore the equilibrium solution of (6.1) is polynomially stable in mean-square.

(iii) Suppose (6.15) holds. Then there exists $\alpha<-1$ such that (6.21) and (6.22) hold. Therefore the equilibrium solution of (6.1) is polynomially stable in mean-square, and a.s. polynomially stable.

Proof. Define $Y(t)=X^{2}(t)$. Itô's rule gives

$$
\begin{aligned}
X^{2}(t)= & X^{2}(0)+\int_{0}^{t} 2 X(s)\left(a X(s)+\sum_{i=1}^{n} b_{i} X\left(q_{i} s\right)\right) d s \\
& +\int_{0}^{t}\left(\sigma X(s)+\sum_{i=1}^{m} \sigma_{i} X\left(r_{i} s\right)\right)^{2} d s \\
& +\int_{0}^{t} 2 X(s)\left(\sigma X(s)+\sum_{i=1}^{m} \sigma_{i} X\left(r_{i} s\right)\right) d B(s) .
\end{aligned}
$$


Let $\left(v_{i}\right)_{i=1, \ldots, n},\left(\lambda_{i}\right)_{i=1, \ldots, m}$ be sequences of positive real numbers. By using the inequalities

$$
2 b_{i} x y \leq\left|b_{i}\right|\left(v_{i}^{2} x^{2}+\frac{1}{v_{i}^{2}} y^{2}\right), \quad 2 \sigma \sigma_{i} x y \leq|\sigma|\left|\sigma_{i}\right|\left(\lambda_{i}^{2} x^{2}+\frac{1}{\lambda_{i}^{2}} y^{2}\right),
$$

for $i=1, \ldots, n, i=1, \ldots, m$ respectively, in conjunction with the inequality proved in Lemma 6.4, we see that (6.23) implies, for any $t, t+h \geq 0$ that

$$
\begin{aligned}
Y(t+h)-Y(t) \leq & \int_{t}^{t+h}\left\{2 a Y(s)+\sum_{i=1}^{n}\left|b_{i}\right|\left(v_{i}^{2} Y(s)+\frac{1}{v_{i}^{2}} Y\left(q_{i} s\right)\right)\right\} d s \\
& +\int_{t}^{t+h}\left\{\sigma^{2} Y(s)+\sum_{i=1}^{m}|\sigma|\left|\sigma_{i}\right|\left(\lambda_{i}^{2} Y(s)+\frac{1}{\lambda_{i}^{2}} Y\left(r_{i} s\right)\right)\right. \\
& \left.\quad+\sum_{l=1}^{m} \sigma_{l}^{2} \mu_{l}^{-2} \cdot \sum_{j=1}^{m} \mu_{j}^{2} Y\left(r_{j} s\right)\right\} d s \\
& +\int_{t}^{t+h} 2 X(s)\left(\sigma X(s)+\sum_{i=1}^{m} \sigma_{i} X\left(r_{i} s\right)\right) d B(s) .
\end{aligned}
$$

As in earlier proofs, by defining $m(t)=\mathbb{E}(|Y(t)|)$, and considering the function $y=y_{v, \lambda, \mu}$ defined by (6.11) with $y(0)>m(0)$, we see that $m(t) \leq y(t)=y_{v, \lambda, \mu}(t)$. By making the appropriate choice of $(v, \lambda, \mu)=\left(v^{*}, \lambda^{*}, \mu^{*}\right)$ in Lemma 6.3, we see that (6.1) is true for some $\alpha \in \mathbb{R}$ if $2 a+\sigma^{2}<0$; for some $\alpha<0$ if (6.14) holds; and for some $\alpha<-1$ if (6.15) holds.

The proof that (6.22) follows from (6.21) differs little from that of the proof of Theorem 4.5, and for this reason is omitted.

We see that the polynomial boundedness of solutions of (6.1) are ensured in mean-square and almost surely if $2 a+\sigma^{2}<0$, so that the terms which involve the delayed arguments do not seem to influence the existence of polynomial asymptotic behaviour for solutions of (6.1). We notice that this mimics the result for solutions of the deterministic pantograph equation with many delays, (6.2).

\subsection{Finite dimensional stochastic pantograph equation}

In [18], Carr and Dyson considered the asymptotic behaviour of the finite dimensional functional differential equation

$$
x^{\prime}(t)=A x(t)+B x(q t),
$$

as $t \rightarrow \infty$. In (6.26), $A, B$ are square matrices, and, for the initial value problem, the solution lies in $C\left(\mathbb{R}^{+} ; \mathbb{R}^{d}\right)$, the space of continuous functions from $\mathbb{R}^{+}$to $\mathbb{R}^{d}$. A consequence of a series of results (Theorems 1-3) in [18] is the following: when all the eigenvalues of $A$ have negative real parts, there exists $\alpha \in \mathbb{R}$ such that

$$
\|x(t)\|=\mathcal{O}\left(t^{\alpha}\right), \quad \text { as } t \rightarrow \infty .
$$

We show that a similar result holds for the equation (6.3), under the hypothesis

All eigenvalues of $A$ have negative real parts.

If the matrix $A$ satisfies (6.27), there exists a positive definite matrix $C$ such that

$$
A^{T} C+C A=-I,
$$


where $A^{T}$ denotes the transpose of the matrix $A$, and $I$ is the $d \times d$ identity matrix. Further, let $\underline{\gamma}^{2}$ and $\bar{\gamma}^{2}$, with $0<\underline{\gamma}^{2} \leq \bar{\gamma}^{2}$, be the minimal and maximal eigenvalues of $C$. Define $V(\bar{x})=\langle x, C x\rangle$, for all $x \in \mathbb{R}^{d}$. By construction $\underline{\gamma}^{2}\|x\|^{2} \leq V(x) \leq \bar{\gamma}^{2}\|x\|^{2}$. Using the CauchySchwarz inequality and the inequality $2 u v \leq \bar{\eta}_{1}^{2} u^{2}+\eta_{1}^{-2} v^{2}$ for $u, v \in \mathbb{R}$, we see for any $x$, $y \in \mathbb{R}^{d}$ that

$$
|\langle x, C B y\rangle+\langle B y, C x\rangle| \leq\left\|B^{T} C\right\|\left(\eta_{1}^{2} \frac{1}{\underline{\gamma}^{2}} V(x)+\frac{1}{\eta_{1}^{2}} \frac{1}{\underline{\gamma}^{2}} V(y)\right) .
$$

Moreover, as

$$
\langle C(\Sigma x+\Theta y), \Sigma x+\Theta y\rangle=\left\langle x, \Sigma^{T} C \Sigma x\right\rangle+\left\langle y, \Theta^{T} C \Theta y\right\rangle+2\left\langle x, \Sigma^{T} C \Theta y\right\rangle,
$$

we can use $2 u v \leq \eta_{2}^{2} u^{2}+\eta_{2}^{-2} v^{2}$ for $u, v \in \mathbb{R}$, to obtain

$$
\begin{aligned}
\langle C(\Sigma x+\Theta y), \Sigma x+\Theta y\rangle \leq \frac{1}{\underline{\gamma}^{2}}\left\|\Sigma^{T} C \Sigma\right\| V(x) & +\frac{1}{\underline{\gamma}^{2}}\left\|\Theta^{T} C \Theta\right\| V(y) \\
& +\left\|\Sigma^{T} C \Theta\right\|\left(\eta_{2}^{2} \frac{1}{\underline{\gamma}^{2}} V(x)+\frac{1}{\eta_{2}^{2}} \frac{1}{\underline{\gamma}^{2}} V(y)\right) .
\end{aligned}
$$

Finally, we have

$$
-\langle x, x\rangle \leq \frac{1}{\bar{\gamma}^{2}} V(x)
$$

Using Itô's rule and (6.28), we have

$$
\begin{aligned}
V(X(t))= & V(X(0))+\int_{0}^{t}-\langle X(s), X(s)\rangle+\langle X(s), C B X(q s)\rangle \\
& +\langle B X(q s), C X(s)\rangle+\langle C(\Sigma X(s)+\Theta X(q s)), \Sigma X(s)+\Theta X(q s)\rangle d s \\
& +\int_{0}^{t}\langle X(s), C(\Sigma X(s)+\Theta X(q s))\rangle+\langle C X(s), \Sigma X(s)+\Theta X(q s)\rangle d B(s) .
\end{aligned}
$$

Putting $m(t)=\mathbb{E}[V(X(t))]$, we proceed as before, using (6.29), (6.30) and (6.31) to show, for any $\varepsilon>0$, that $m(t) \leq y(t)$, where we have $y(0)=(1+\varepsilon) m(0)>m(0)$, and

$$
\begin{aligned}
y^{\prime}(t)=\left(-\frac{1}{\bar{\gamma}^{2}}+\frac{\left\|B^{T} C\right\|}{\underline{\gamma}^{2}} \eta_{1}^{2}+\frac{1}{\underline{\gamma}^{2}}\right. & \left.\left\|\Sigma^{T} C \Sigma\right\|+\frac{1}{\underline{\gamma}^{2}}\left\|\Sigma^{T} C \Theta\right\| \eta_{2}^{2}\right) y(t) \\
& +\left(\frac{\left\|B^{T} C\right\|}{\underline{\gamma}^{2}} \frac{1}{\eta_{1}^{2}}+\frac{1}{\underline{\gamma}^{2}}\left\|\Theta^{T} C \Theta\right\|+\frac{1}{\underline{\gamma}^{2}}\left\|\Sigma^{T} C \Theta\right\| \frac{1}{\eta_{2}^{2}}\right) y(q t) .
\end{aligned}
$$

Notice moreover that we also have $m(0)=\mathbb{E}[\langle X(0), C X(0)\rangle] \leq\|C\| \mathbb{E}\left[\left\|X_{0}\right\|^{2}\right]$. Thus

$$
\mathbb{E}\left[\|X(t)\|^{2}\right] \leq \frac{1}{\underline{\gamma}^{2}} m(t) \leq \frac{1}{\underline{\gamma}^{2}} y(t) .
$$

We are now in a position to distill the result of this discussion into the following theorem.

Theorem 6.6. Let $(X(t))_{t \geq 0}$ be the solution of (6.3) with $\mathbb{E}\left(\left\|X_{0}\right\|^{4}\right)<\infty$. Suppose that (6.27) holds. If $\underline{\gamma}^{2}$ and $\bar{\gamma}^{2}$, with $0<\underline{\gamma}^{2} \leq \bar{\gamma}^{2}$, are the maximum and minimum eigenvalues of the positive definite matrix $C$ which satisfies $A^{T} C+C A=-I$, then the following holds. 
(i) Suppose

$$
\frac{1}{\bar{\gamma}^{2}}+\frac{1}{\underline{\gamma}^{2}}\left\|\Sigma^{T} C \Sigma\right\|<0
$$

Then there exist $\alpha \in \mathbb{R}$ and $C>0$ such that

$$
\mathbb{E}\left(\|X(t)\|^{2}\right) \leq C \mathbb{E}\left[\|X(0)\|^{2}\right] t^{\alpha},
$$

and

$$
\limsup _{t \rightarrow \infty} \frac{\log \|X(t)\|}{\log t} \leq \frac{1}{2}(\alpha+1), \quad \text { a.s. }
$$

Therefore, the process is globally polynomially bounded in mean-square and is almost surely globally polynomially bounded.

(ii) Suppose

$$
-\frac{1}{\bar{\gamma}^{2}}+\frac{1}{\underline{\gamma}^{2}}\left(\left\|\Sigma^{T} C \Sigma\right\|+\left\|\Theta^{T} C \Theta\right\|+2\left\|B^{T} C\right\|+2\left\|\Sigma^{T} C \Theta\right\|\right)<0 .
$$

Then there exists $\alpha<0$ such that (6.32) and (6.33) hold. Therefore the equilibrium solution of (6.3) is globally polynomially stable in mean-square, and the solution process of (6.3) is almost surely globally polynomially bounded.

(iii) Suppose

$$
-\frac{1}{\bar{\gamma}^{2}}+\frac{1}{\underline{\gamma}^{2}}\left(\left\|\Sigma^{T} C \Sigma\right\|+\frac{\left\|\Theta^{T} C \Theta\right\|}{q}+\frac{2}{\sqrt{q}}\left\|B^{T} C\right\|+\frac{2}{\sqrt{q}}\left\|\Sigma^{T} C \Theta\right\|\right)<0 .
$$

Then there exists $\alpha<-1$ such that (6.32) and (6.33) hold. Therefore the equilibrium solution of (6.3) is globally polynomially stable in mean-square, and a.s. globally polynomially stable.

We note that we can obtain weaker sufficient conditions under which the conclusions of Theorem 6.6 hold, which can be more easily interpreted and verified. Instead of (6.29) and (6.30), we have

$$
|\langle x, C B y\rangle+\langle B y, C x\rangle| \leq\|B\|\|C\|\left(\eta_{1}^{2} \frac{1}{\underline{\gamma}^{2}} V(x)+\frac{1}{\eta_{1}^{2}} \frac{1}{\underline{\gamma}^{2}} V(y)\right)
$$

and

$$
\begin{aligned}
\langle C(\Sigma x+\Theta y), \Sigma x+\Theta y\rangle \leq \frac{1}{\underline{\gamma}^{2}}\|\Sigma\|^{2}\|C\| V(x) & +\frac{1}{\underline{\gamma}^{2}}\|\Theta\|^{2}\|C\| V(y) \\
& +\|\Sigma\|\|C\|\|\Theta\|\left(\eta_{2}^{2} \frac{1}{\underline{\gamma}^{2}} V(x)+\frac{1}{\eta_{2}^{2}} \frac{1}{\underline{\gamma}^{2}} V(y)\right) .
\end{aligned}
$$

Since $C$ is symmetric, $\|C\|=\bar{\gamma}^{2}$. Using these estimates in the proof of Theorem 6.6 gives the following result.

Corollary 6.7. Let $(X(t))_{t \geq 0}$ be the solution of (6.3) with $\mathbb{E}\left(\left\|X_{0}\right\|^{4}\right)<\infty$. Suppose that (6.27) holds. If $\underline{\gamma}^{2}$ and $\bar{\gamma}^{2}$, with $0<\underline{\gamma}^{2} \leq \bar{\gamma}^{2}$, are the maximum and minimum eigenvalues of the positive definite matrix $C$ which satisfies $A^{T} C+C A=-I$, then the following holds: 
(i) Suppose

$$
\frac{\bar{\gamma}^{2}}{\bar{\gamma}^{4}}>\|\Sigma\|^{2}
$$

Then there exist $\alpha \in \mathbb{R}, C>0$ such that (6.32) and (6.33) hold.

(ii) Suppose

$$
\frac{\bar{\gamma}^{2}}{\bar{\gamma}^{4}}>(\|\Sigma\|+\|\Theta\|)^{2}
$$

Then there exists $\alpha<0$ such that (6.32) and (6.33) hold.

(iii) Suppose

$$
\frac{\underline{\gamma}^{2}}{\bar{\gamma}^{4}}>\frac{2}{\sqrt{q}}\|B\|+\left(\|\Sigma\|+\frac{\|\Theta\|}{\sqrt{q}}\right)^{2} .
$$

Then there exists $\alpha<-1$ such that (6.32) and (6.33) hold.

Therefore, if the intensities of the noise terms are sufficiently small, the negative spectrum of $A$ ensures the polynomial asymptotic stability of the noise perturbed system, thereby following the global polynomial asymptotic stability exhibited for (6.26). Note once again that the polynomial mean square and almost sure bounds on the solution exist, provided the intensity of noise from the non-delay term is sufficiently small and $A$ has a negative spectrum: as is the case for equation (6.26), the presence of polynomial asymptotic behaviour does not seem to be determined by the terms with delayed arguments.

\section{Concluding remarks}

There are a number of related problems which we have not studied in this paper, which nonetheless can be treated using the analysis presented here. For instance, all the stochastic evolutions we have considered above are driven by a single Brownian motion, but no new ideas are required to extend the results to stochastic functional differential equations driven by finitely many Brownian motions. Another interesting class of equations to study are the stochastic analogues of the pantograph equations studied in, e.g. [30,38], where the delayed argument is not necessarily of proportional form, and the rate of decay or growth of solutions is not necessarily polynomially fast. We hope to consider the asymptotic behaviour of such stochastic equations elsewhere. We have also omitted to study nonlinear and nonautonomous versions of the stochastic pantograph equation, for example

$$
d X(t)=\left(f_{1}(t, X(t))+g_{1}(t, X(q t))\right) d t+\left(f_{2}(t, X(t))+g_{2}(t, X(q t))\right) d B(t),
$$

where $f_{1}, f_{2}$ are globally linearly bounded and Lipschitz continuous. If however, the function $f_{1}$ satisfies

$$
\left\langle f_{1}(t, x), x\right\rangle \leq-a\|x\|^{2}
$$

for all $x \in \mathbb{R}, t \geq 0$ and some $a>0$, we can again recover the polynomial asymptotic behaviour exhibited by the processes studied in this paper for this process. Finite dimensional and many-delay analogues of the extensions mentioned here can be treated using the techniques of the previous section. 


\section{Appendix}

We will require some further supporting results from stochastic analysis, and elementary properties of the stochastic pantograph equation.

Before we give the proof of Lemma 3.4, which was earlier deferred, we first recall for a continuous real-valued function $f$ of a real variable, the Dini derivative $D_{-} f$ is defined as

$$
D_{-} f(t)=\liminf _{\delta \uparrow 0} \frac{f(t+\delta)-f(t)}{\delta} .
$$

To prove Lemma 3.4, we also require the following result, which appears as Lakshmikantham and Leela [31, Vol. 1, Lemma 1.2.2].

Lemma 8.1. Let $v, w$ be continuous functions and $D v(t) \leq w(t)$ for $t$ in an interval with a possible exceptional set of measure zero and $D$ being a fixed Dini derivative. Then $D_{-} v(t) \leq w(t)$ holds for $t$ in an interval, with a possible exceptional set of measure zero.

We now turn to the proof of Lemma 3.4.

Proof. With $x$ defined by (3.5) and $p$ defined by (3.6) with $0<p(0)<x(0)$, we will show that

$$
p(t)<x(t)
$$

for all $t \geq 0$. Assume that (8.1) is false; then there exists $T>0$ such that the set

$$
Z=\{t \in[0, T): p(t) \geq x(t)\}
$$

is nonempty. We set $t_{1}=\inf Z$ and as $p(0)<x(0)$, we have that $0<t_{1}$ and

$$
p\left(t_{1}\right)=x\left(t_{1}\right) \text { and } p(t)<x(t) \text { for } t \in\left[0, t_{1}\right) .
$$

Then, for all $-\left|t_{1}\right|<h<0$, as $p\left(t_{1}+h\right)<x\left(t_{1}+h\right)$,

$$
\frac{1}{h}\left\{\left(p\left(t_{1}+h\right)-\left(p\left(t_{1}\right)\right\}>\frac{1}{h}\left\{x\left(t_{1}+h\right)\right)-x\left(t_{1}\right)\right)\right\},
$$

so letting $h \uparrow 0$, we get

$$
D_{-} p\left(t_{1}\right) \geq x^{\prime}\left(t_{1}\right)
$$

Next by Lemma 8.1, as $p$ obeys (3.6) and $t \mapsto \bar{a} p(t)+\bar{b} p(q t)$ is continuous, we have

$$
D_{-} p(t) \leq \bar{a} p(t)+\bar{b} p(q t), \quad t>0 .
$$

In particular, this yields

$$
D_{-} p\left(t_{1}\right) \leq \bar{a} p\left(t_{1}\right)+\bar{b} p\left(q t_{1}\right) .
$$

Thus, (8.2), (8.3) and (3.5), together with the facts that $p\left(t_{1}\right)=x\left(t_{1}\right)$, and $p\left(q t_{1}\right)<x\left(q t_{1}\right)$, imply

$$
\begin{aligned}
0 & \leq D_{-} p\left(t_{1}\right)-x^{\prime}\left(t_{1}\right) \\
& \leq \bar{a} p\left(t_{1}\right)+\bar{b} p\left(q t_{1}\right)-\left(\bar{a} x\left(t_{1}\right)+\bar{b} x\left(q t_{1}\right)\right) \\
& =\bar{b}\left(p\left(q t_{1}\right)-x\left(q t_{1}\right)\right) \\
& <0
\end{aligned}
$$

which is a contradiction. The result therefore follows. 
The following result is due to LeGall [32].

Lemma 8.2. Suppose $X$ is a continuous semi-martingale with decomposition

$$
X(t)=X_{0}+V(t)+M(t)
$$

where $M$ is a continuous local martingale and $V$ is the difference of continuous, non-decreasing adapted processes with $V_{0}=0$, a.s. If $k:(0, \infty) \rightarrow(0, \infty)$ is a Borel function satisfying

$$
\int_{0}^{\varepsilon} \frac{1}{k(x)} d x=\infty \quad \text { for all } \varepsilon>0
$$

and

$$
\int_{0}^{t} \frac{d\langle M\rangle(s)}{k(X(s))} 1_{\{X(s)>0\}} d s<\infty \quad \text { for all } t \text { a.s., }
$$

then the semi-martingale local time of $X$ at 0 is identically 0 , almost surely.

Remark 8.3. For the equation (2.1) with $\rho=0$ i.e.,

$$
d X(t)=(a X(t)+b X(q t)) d t+\sigma X(t) d B(t),
$$

note that $k(x)=x^{2}$ satisfies the conditions of Lemma 8.2.

In the next Lemma, we extend results on moment bounds for the stochastic pantograph equation from [14].

Lemma 8.4. If $X\left(t ; 0, X_{0}\right)$ is a solution of (2.1) with $\mathbb{E}\left(X_{0}^{4}\right)<\infty$, then

(i) $t \mapsto \mathbb{E}\left(|X(t)|^{m}\right)$ is continuous on $\mathbb{R}^{+}$for $m=1,2$,

(ii) $\mathbb{E} \int_{0}^{t}|X(s)|^{2 m} d s<\infty$ for all fixed $t \geq 0$ and for $m=1,2$.

Proof. In [14] it is shown, that $\mathbb{E}\left(|X(t)-X(s)|^{2}\right) \leq C(T) \cdot(t-s)$ for any $0 \leq s \leq t \leq T$ with $t-s<1$. Part (i) for $m=1$ now follows from the inequalities:

$$
\begin{aligned}
|\mathbb{E}(|X(t)|)-\mathbb{E}(|X(s)|)| & \leq \mathbb{E} \| X(t)|-| X(s)|| \leq \mathbb{E}(|X(t)-X(s)|) \\
& \leq\left(\mathbb{E}(X(t)-X(s))^{2}\right)^{\frac{1}{2}} .
\end{aligned}
$$

For Part (i) for $m=2$ we have:

$$
\begin{aligned}
\left|\mathbb{E}\left(X^{2}(t)\right)-\mathbb{E}\left(X^{2}(s)\right)\right| & =\left|\mathbb{E}\left(X^{2}(t)-X^{2}(s)\right)\right| \\
& \leq \mathbb{E}\left[\left|X^{2}(t)-X^{2}(s)\right|\right] \\
& \leq \mathbb{E}[|(X(t)-X(s))(X(t)+X(s))|] \\
& \leq \mathbb{E}|X(t)-X(s)|^{2} \mathbb{E}|X(t)+X(s)|^{2} .
\end{aligned}
$$

Now, $t \mapsto \mathbb{E}\left(\sup _{0 \leq s \leq t}|X(s)|^{2}\right)$ is bounded on compact sets, [14, Theorem 2.3], so applying this proves (i) for $m=2$.

To establish (ii), note that, for $m=1$, it follows immediately from the last statement. Modifying the argument of [14, Theorem 2.3] establishes that the function $t \mapsto \mathbb{E}\left(\sup _{0 \leq s \leq t}|X(s)|^{4}\right)$ is bounded, which proves (ii) for $m=2$. 


\section{Acknowledgements}

The authors were supported by a SQuaRE activity entitled "Stochastic stabilisation of limitcycle dynamics in ecology and neuroscience" funded by the American Institute of Mathematics.

The authors are pleased to thank the organisers of the conference and the editors of the proceedings for the opportunity to submit their work to its contents. The authors are also extremely grateful to the referee for their very careful reading and correction of the paper, and especially the referee's criticism of the definitions of stability and boundedness. This criticism has lead to an improved formulation of the definitions and associated results.

\section{References}

[1] J. A. D. Appleby, $p^{\text {th }}$ mean integrability and almost sure asymptotic stability of ItôVolterra equations, J. Integral Equations Appl. 15(2003), No. 4, 321-341. MR2058807

[2] J. A. D. Appleby, Subexponential solutions of scalar linear Itô-Volterra equations with damped stochastic perturbations, Funct. Differ. Equ. 11(2004), Nos. 1-2, 5-10. MR2054754

[3] J. A. D. Appleby, Decay and growth rates of solutions of scalar stochastic delay differential equations with unbounded delay and state dependent noise, Stoch. Dyn. 5(2005), No. 2, 133-148. MR2147278

[4] J. A. D. Appleby, Almost sure subexponential decay rates of scalar Itô-Volterra equations, Electron. J. Qual. Theory Differ. Equ., Proc. 9th Coll. QTDE 2012, No. 1, 1-32. MR2170469

[5] J. A. D. Appleby, E. Buckwar, Sufficient condition for polynomial asymptotic behaviour of stochastic pantograph equations, preprint, 2003. url

[6] J. A. D. Appleby, E. Buckwar, A constructive comparison technique for determining the asymptotic behaviour of linear functional differential equations with unbounded delay, Differ. Equ. Dynam. Syst. 18(2010), No. 3, 271-301. MR2770537

[7] J. A. D. Appleby, D. Mackey, Polynomial asymptotic stability of damped stochastic differential equations, Electron. J. Qual. Theory Differ. Equ., Proc. 7th Coll. QTDE 2004, No. 2, 1-33. MR2170470

[8] J. A. D. Appleby, D. D. Patterson, On necessary and sufficient conditions for preserving convergence rates to equilibrium in deterministically and stochastically perturbed differential equations with regularly varying nonlinearity, in: Recent advances in delay differential and difference Equations, Springer Proceedings in Mathematics and Statistics, Vol. 94, eds. M. Pituk, F. Hartung, Springer, Cham, 2014, 1-85. MR3280185

[9] J. A. D. Appleby, D. W. Reynolds, Subexponential solutions of linear Volterra integrodifferential equations and transient renewal equations, Proc. Roy. Soc. Edinburgh Sect. A 132(2002), 521-543. MR1912414

[10] J. A. D. Appleby, D. W. Reynolds, Non-exponential stability of scalar stochastic Volterra equations, Statist. Probab. Lett. 62(2003), No. 4, 335-343. MR1973308 
[11] J. A. D. Appleby, D. W. Reynolds, Decay rates of solutions of linear stochastic Volterra equations, Electron. J. Probab. 13(2008), No. 30, 922-943. MR2413289

[12] J. A. D. Appleby, M. Riedle, Stochastic Volterra differential equations in weighted spaces, J. Integral Equ. Appl. 22(2010), No. 1, 1-17. MR2607553

[13] C. T. H. BAKER, E. BuCKWAR, Exponential stability in $p$-th mean of solutions, and of convergent Euler-type solutions, of stochastic delay differential equations, J. Comput. Appl. Math. 184(2005), No. 2, 404-427. MR2157336

[14] C. T. H. BAKer, E. Buckwar, Continuous $\theta$-methods for the stochastic pantograph equation, Electron. Trans. Numer. Anal. 11(2000), 131-151. MR1799028

[15] H. Brunner, P. J. van DER Houwen, The numerical solution of Volterra equations, CWI Monographs, Vol. 3, North-Holland, Amsterdam, 1986. MR0871871

[16] T. Caraballo, On the decay rate of solutions of non-autonomous differential systems, Electron. J. Differential Equations 2001, No. 5, 1-17. MR1811778

[17] J. CARR, J. Dyson, The functional differential equation $y^{\prime}(x)=a y(\lambda x)+b y(x)$, Proc. Roy. Soc. Edinburgh Sect. A 74(1974/5), 165-174. MR0442421

[18] J. CARR, J. Dyson, The matrix functional differential equation $y^{\prime}(x)=A y(\lambda x)+B y(x)$, Proc. Roy. Soc. Edinburgh Sect. A 75(1975/6), No. 1, 5-22. MR0442422

[19] Z. FAN, M. LiU, W. CAO, Existence and uniqueness of the solutions and convergence of semi-implicit Euler methods for stochastic pantograph equations, J. Math. Anal. Appl. 325(2007), No. 2, 1142-1159. MR2270075

[20] L. Fox, D. F. Mayers, J. R. Ockendon, A. B. TAyler, On a functional differential equation, J. Inst. Maths. Appl. 8(1971), 271-307. MR0301330

[21] J. R. Haddock, T. Krisztin, Estimates regarding the decay of solutions of functional differential equations, Nonlinear Anal. 8(1984), No. 12, 1395-1408. MR0769402

[22] J. R. HADDOcK, T. KRIszTin, On the rate of decay of solutions of functional differential equations with infinite delay, Nonlinear Anal. 10(1986), No. 8, 727-742. MR0851143

[23] Y. Hu, F. Wu, C. HuANG, Stochastic stability of a class of unbounded delay neutral stochastic differential equations with general decay rate, Internat. J. Systems Sci. 43(2012), No. 2, 308-318. MR2862245

[24] Y. HuAnG, Z. YAN, H. ZHENG, Stochastic $H_{\infty}$ filtering for pantograph systems with statedependent noise, in: 2012 IEEE International Conference on Automation and Logistics (ICAL), 5-17 August 2012, Zhengzhou, 2012, pp. 601-605. url

[25] A. IserLes, On the generalized pantograph functional-differential equation, European J. Appl. Math. 4(1993), No. 1, 1-38. MR1208418

[26] A. IserLes, Exact and discretized stability of the pantograph equation, Appl. Numer. Math. 24(1997), 295-308. MR1464730; url 
[27] F. JIAng, H. YANG, S. WANG, Asymptotic stability of stochastic pantograph differential equations with Markovian switching, J. Nonlinear Anal. Optim. 1(2010), No. 1, 9-16. MR2911682

[28] I. Karatzas, S. E. Shreve, Brownian motion and stochastic calculus, second edition, Graduate Texts in Mathematics, Vol. 113, Springer, New York, 1991. MR1121940

[29] T. Kato, J. B. McLeod, The functional-differential equation $y^{\prime}(x)=a y(\lambda x)+b y(x), B u l l$. Amer. Math. Soc. 77(1971), 891-937. MR0283338

[30] T. KRIszTIN, On the convergence of solutions of functional differential equations with infinite delay, J. Math. Anal. Appl. 109(1985), No. 2, 509-521. MR0802910

[31] V. Lakshmikantham, S. Leela, Differential and integral inequalities: Theory and applications. Vol I: Ordinary differential equations, Mathematics in Science and Engineering, Vol. 55-I, Academic Press, New York, 1969. MR0379933

[32] J.-F. LeGall, Applications du temps locaux aux équations différentielles stochastiques unidimensionnelles (in French) [Local time applications to one-dimensional stochastic differential equations], Seminar on probability, XVII, 15-31, Lecture Notes in Math., Vol. 986, Springer, Berlin, 1983 MR0770393

[33] W. LiU, M. Foondun, X. MaO, Mean square polynomial stability of numerical solutions to a class of stochastic differential equations, Statist. Probab. Letters 92(2014), 173-182. MR3230491

[34] K. Liv, Some remarks on exponential stability of stochastic differential equations, Stochastic Anal. Appl. 19(2001), No. 1, 59-65. MR1814199

[35] K. LiU, X. MAO, Large time decay behavior of dynamical equations with random perturbation features, Stochastic Anal. Appl. 19(2001), No. 2, 295-327. MR1841441

[36] X. LIU, T. CHEN, Robust $\mu$-stability for uncertain stochastic neural networks with unbounded time-varying delays, Phys. A. 387(2008), No. 12, 2952-2962. MR2587192

[37] Y. LIU, On functional differential equations with proportional delays, Ph.D. thesis, Department of Mathematics, Cambridge University, Cambridge, 1996.

[38] G. MaKAY, J. TERJÉKI, On the asymptotic behavior of the pantograph equations, Electron. J. Qual. Theory Differ. Equ. 1998, No. 2, 1-12. MR1615106

[39] X. MaO, Almost sure asymptotic bounds for a class of stochastic differential equations, Stochastics Stochastics Rep. 41(1992), No. 1-2, 57-69. MR1275366

[40] X. MAO, Almost sure polynomial stability for a class of stochastic differential equations, Quart. J. Math. Oxford Ser. (2) 43(1992), No. 171, 339-348. MR1176492

[41] X. MAO, Polynomial stability for perturbed stochastic differential equations with respect to semimartingales, Stochastic Process. Appl. 41(1992), No. 1, 101-116. MR1162721

[42] X. MAo, Exponential stability of stochastic differential equations, Monographs and Textbooks in Pure and Applied Mathematics, Vol. 182, Marcel Dekker, New York, 1994. MR1275834 
[43] X. Mao, Stochastic differential equations and their applications, Horwood Publishing Limited, Chichester, 1997. MR1475218

[44] X. Meng, S. Hu, P. Wu, Pathwise estimation of stochastic differential equations with unbounded delay and its application to stochastic pantograph equations, Acta Appl. Math. 113(2011), No. 2, 231-246. MR2763306

[45] X. MenG, B. Yin, On the general decay stability of stochastic differential equations with unbounded delay, J. Korean Math. Soc. 49(2012), No. 3, 515-536. MR2953034

[46] S. Murakami, Exponential asymptotic stability for scalar linear Volterra equations, Differential Integral Equations 4(1991), No. 3, 519-525. MR1097915

[47] J. R. Ockendon, A. B. TAYLER, The dynamics of a current collection system for an electric locomotive, Proc. R. Soc. A 322(1971), 447-468. url

[48] L. Ronghua, L. Min, P. Wan-KaI, Convergence of numerical solutions to stochastic pantograph equations with Markovian switching, Appl. Math. Comput. 215(2009), No. 1, 414-422. MR2568343

[49] M. H. Song, Y. L. Lu, M. Z. LiU, Stability of analytical solutions and convergence of numerical methods for non-linear stochastic pantograph differential equations, $12 \mathrm{pp}$, 2015. Available on arXiv.

[50] Z. YAN AND Y. HuANG, Robust $H_{\infty}$ filter design for Itô stochastic pantograph systems, Mathe. Probl. Eng. 2013(2013), Art. ID 747890, 8 pp. MR3044306

[51] H. YANG, F. JIANG, Y. JIANG, Robust stability of stochastic pantograph differential equations Markovian switching, in: International Conference on Computer Application and System Modelling (ICCASM), October 22-24, 2010, Shanxi, Taiyuan, Vol. 5, pp. 541-545, 2010. url

[52] H. YANG, F. JIANG, Stochastic $\theta$-methods for a class of jump-diffusion stochastic pantograph equations with random magnitude, Scientific World J. 2014, Art. ID 589167, 9 pp. url

[53] B. Zhang, A. H. Tsor, Lyapunov functions in weak exponential stability and controlled stochastic systems, J. Ramanujan Math. Soc 11(1996), No. 2, 85-102. MR1429302

[54] B. Zhang, A. H. Tsoi, Weak exponential stability of stochastic differential equations, Stochastic Anal. Appl. 15(1997), No. 4, 643-649. MR1464410

[55] H. Zhang, Mean square convergence of semi-implicit Euler methods for nonlinear neutral stochastic pantograph equations, Int. J. Numer. Methods Appl. 12(2014), No. 2, 109-132. MR3329662

[56] H. Zhang, Y. XIAO, F. Guo, Convergence and stability of a numerical method for nonlinear stochastic pantograph equations, J. Franklin Inst. 351(2014), No. 6, 3089-3103. MR3201021 